\title{
S-nitrosylation of connexin43 hemichannels elicits cardiac stress- induced arrhythmias in Duchenne muscular dystrophy mice
}

\author{
Mauricio A. Lillo, ${ }^{1}$ Eric Himelman, ${ }^{2}$ Natalia Shirokova, ${ }^{1}$ Lai-Hua Xie, ${ }^{2}$ Diego Fraidenraich, ${ }^{2}$ \\ and Jorge E. Contreras' \\ 'Department of Pharmacology, Physiology and Neuroscience and 'Department of Cell Biology and Molecular Medicine, \\ Rutgers New Jersey Medical School, Newark, New Jersey, USA.
}

Patients with Duchenne muscular dystrophy (DMD) commonly present with severe ventricular arrhythmias that contribute to heart failure. Arrhythmias and lethality are also consistently observed in adult $D m d^{m d x}$ mice, a mouse model of DMD, after acute $\beta$-adrenergic stimulation. These pathological features were previously linked to aberrant expression and remodeling of the cardiac gap junction protein connexin43 (Cx43). Here, we report that remodeled $C_{x 43}$ protein forms $C \times 43$ hemichannels in the lateral membrane of $D m d^{m d x}$ cardiomyocytes and that the $\beta$-adrenergic agonist isoproterenol (Iso) aberrantly activates these hemichannels. Block of Cx43 hemichannels or a reduction in Cx43 levels (using $D m d^{m d x} \mathrm{Cx}_{43^{+/-}}$mice) prevents the abnormal increase in membrane permeability, plasma membrane depolarization, and Iso-evoked electrical activity in these cells. Additionally, Iso treatment promotes nitric oxide (NO) production and S-nitrosylation of Cx43 hemichannels in $D m d^{m d x}$ heart. Importantly, inhibition of NO production prevents arrhythmias evoked by Iso. We found that NO directly activates Cx43 hemichannels by S-nitrosylation of cysteine at position 271. Our results demonstrate that opening of remodeled and S-nitrosylated Cx43 hemichannels plays a key role in the development of arrhythmias in DMD mice and that these channels may serve as therapeutic targets to prevent fatal arrhythmias in patients with DMD .

Conflict of interest: The authors have declared that no conflict of interest exists.

Copyright: (c) 2019, American Society for Clinical Investigation.

Submitted: May 7, 2019

Accepted: November 7, 2019

Published: November 12, 2019

Reference information: /CI Insight. 2019;4(24):e130091.

https://doi.org/10.1172/jci.

insight.130091.

\section{Introduction}

Duchenne muscular dystrophy (DMD) is an incurable, progressive muscle disease that affects approximately 1 in 3500 male births (1). DMD is caused by lack of the functional structural protein dystrophin. Absence of dystrophin increases cellular fragility, resulting in recurrent skeletal and cardiac muscle damage during contraction (2). Currently, steroid therapy and assisted ventilation help combat skeletal muscle-related respiratory dysfunction and significantly prolong the lives of DMD patients. However, cardiac dysfunction (e.g., reduced contractility and arrhythmias) is becoming a prominent contributor to DMD pathology $(3,4)$, and over $40 \%$ of patients die from heart failure $(1,5)$.

Current treatments to attenuate DMD cardiomyopathies rely mainly on angiotensin-converting enzyme inhibitors, $\beta$-adrenergic blockers, and avoidance of high-intensity, adrenaline-inducing activities $(6,7)$. Indeed, $\beta$-adrenergic signaling is highly dysregulated in DMD hearts. For example, cardiac stress induces oxidative stress and hyperactivity of $\mathrm{Ca}^{2+} /$ calmodulin-dependent protein kinase II and ryanodine receptor 2 (RyR2), which triggers ventricular arrhythmias in young mutant X-linked dystrophin gene mice ( $\left.D m d^{m d x}\right)$ mice $(8,9)$. At later stages of the disease, "leaky" RyR2 channels contribute to aberrant $\mathrm{Ca}^{2+}$ release from the sarcoplasmic reticulum. This $\mathrm{Ca}^{2+}$ leak triggers a signaling pathway that promotes plasma membrane potential $(V m)$ depolarization and, consequently, delayed after-depolarizations (DADs) (10). However, plasma membrane channels/transporters responsible for changes in $V m$ in $D m d^{m d x}$ cardiomyocytes remain elusive.

In the heart, cardiomyocytes are arranged laterally end to end and connected through intercalated discs. The intercalated discs of healthy cardiomyocytes contain gap junctions, which act as low-resistance channels to an opposing cardiomyocyte (11). Connexin $43(\mathrm{Cx} 43)$ is the most abundant connexin isoform and is found in the working myocardium of the atrium and ventricle as well as in the more distal regions 
of the Purkinje network (12). The biogenesis of a $\mathrm{Cx} 43$ gap junction channel begins with the intracellular assembly of 6 connexin ( $\mathrm{Cx}$ ) proteins to form a hemichannel, which is then inserted into the plasma membrane. The hemichannel moves to sites of apposition between cells and docks with a hemichannel of an adjacent cell to form a gap junction channel. Importantly, myocytes from diseased hearts display abnormal levels of $\mathrm{Cx} 43$ and redistribute to the plasma membrane away from the intercalated discs. This increase of Cx43 in lateralized regions of diseased cardiomyocytes is a phenomenon known as remodeling (13-15). Cx43 remodeling is observed in several pathological cardiac conditions, including ischemia, hypertrophy, and heart failure, as well as in DMD (13-17).

We have recently proposed that in the heart, remodeled $\mathrm{Cx} 43$ proteins at the plasma membrane in $D m d^{m d x}$ mice do not form gap junctions, but instead, undocked hemichannels $(16,18)$. Thus we hypothesize that $\beta$-adrenergic stimulation enhances the activity of remodeled Cx43 hemichannels in Dmd ${ }^{m d x}$ hearts, affecting cardiomyocyte membrane excitability and promoting arrhythmias. Here, we tested this idea and demonstrated that $\beta$-adrenergic stimulation leads to the opening of $\mathrm{Cx} 43$ hemichannels via nitric oxide (NO) production and direct S-nitrosylation of Cx43 proteins. Inhibition of NO synthesis prevented S-nitrosylation of $\mathrm{Cx} 43$ and arrhythmias evoked by $\beta$-adrenergic stimulation in $D m d^{m d x}$ mice. Consistent with this observation, S-nitrosylation of $\mathrm{Cx} 43$ hemichannels resulted in membrane plasma depolarization of $D m d^{m d x}$ cardiomyocytes and subsequent generation of action potentials. Finally, we determined that $\mathrm{Cx} 43$ hemichannel activity increased after S-nitrosylation of cysteine 271 in the C-terminal domain. We propose that enhanced S-nitrosylation and opening of remodeled Cx43 hemichannels are critical for the development of arrhythmias in DMD.

\section{Results}

Isoproterenol-evoked electrical activity in Dmdindx cardiomyocytes is mediated by Cx43 hemichannels. We tested the hypothesis that lateralized $\mathrm{Cx} 43$ protein forms hemichannels with aberrant activity, which results in increased membrane excitability and favors isoproterenol-induced (Iso-induced) arrhythmias in $D m d^{m d x}$ cardiomyocytes. Figure 1A shows representative traces of cardiac action potentials (APs) from WT and Dmd ${ }^{m d x}$ isolated cardiomyocytes evoked by an injection of $2 \mathrm{nA}$ current under current-clamp conditions. Treatment of cells with $1 \mu \mathrm{M}$ Iso induced triggered activity (TA) in $D m d^{m d x}$, but not WT, cardiomyocytes. The average number of Iso-induced TAs was $63 \pm 1.8$ and $2 \pm 0.5$ per minute in $D m d^{m d x}$ and WT cardiomyocytes, respectively (Figure 1B).

To assess the role of $\mathrm{Cx} 43$ hemichannels in Iso-evoked TA, we examined whether the latter is prevented by 2 specific Cx43 hemichannel blockers added into the patch pipette: Gap19 peptide $(19,20)(232 \mathrm{ng} /$ $\mu \mathrm{L})$ or $\mathrm{AbCx} 43$ antibody $(2.5 \mathrm{ng} / \mu \mathrm{L})$ that recognizes the C-terminal $(\mathrm{CT})$ domain of $\mathrm{Cx} 43$. Iso-evoked TAs were significantly reduced (by $\sim 80 \%$ ) in $D m d^{m d x}$ cardiomyocytes treated with both Gap19 peptide (8.2 \pm 0.6 per minute) and $\mathrm{AbCx} 43$ (7.4 \pm 0.4 per minute) (Figure $1, \mathrm{~A}$ and $\mathrm{B}$ ). Importantly, blockade of $\mathrm{Cx} 45$ hemichannels, a distinct $\mathrm{Cx}$ isoform also expressed in cardiomyocytes (12), with an antibody against the Cx45 CT domain, did not prevent the generation of TA (Figure 1, A and B).

Previously, we showed that dystrophic mice with lower levels of $\mathrm{Cx} 43\left(D m d^{m d x} \mathrm{Cx} 43^{+/-}\right)$were resistant to Iso-induced arrhythmias (18). $D m d^{m d x} \mathrm{Cx} 43^{+/-}$cardiomyocytes display less lateralized $\mathrm{Cx} 43$ protein and likely reduced hemichannel activity (18). We asked, therefore, whether dystrophic cardiomyocytes from $D m d^{m d x}$ $\mathrm{Cx} 43^{+/-}$mice were less susceptible to Iso-induced TAs. Upon treatment with $1 \mu \mathrm{M}$ Iso, isolated cardiac cells from $D m d^{m d x} \mathrm{Cx} 43^{+/-}$displayed a substantial reduction (by $~ 70 \%$ ) of TAs compared with Dmd ${ }^{m d x}$ cardiomyocytes. Addition of the $\mathrm{AbCx} 43$ antibody in the pipette completely prevented TAs in $D m d^{m d x} \mathrm{Cx} 43^{+/-}$cardiomyocytes (Figure 1A). The average number of TAs was $18.4 \pm 3.2$ and $3.2 \pm 0.8$ per minute in $D m d^{m d x} \mathrm{Cx} 43^{+/-}$in the absence and presence of $\mathrm{AbCx} 43$, respectively (Figure 1B). These data strongly suggest that Iso-evoked TAs in $D m d^{m d x}$ cardiomyocytes are mediated by activity of Cx43 hemichannels.

We next examined whether Iso-induced TAs in $D m d^{m d x}$ cardiomyocytes are associated with changes in the resting $V m$, which in turn are caused by the altered activity of Cx43 hemichannels. Figure $1 \mathrm{C}$ shows that $D m d^{m d x}$ cardiomyocytes are more depolarized with respect to WT cardiomyocytes under normal conditions, with $V m$ values of $-65.3 \pm 2.1 \mathrm{mV}$ and $-67.8 \pm 3.2 \mathrm{mV}$, respectively. Iso stimulation further depolarized both $D m d^{m d x}$ and WT cardiomyocytes to $V m$ values of $-61.4 \pm 1.3 \mathrm{mV}$ and $-64.6 \pm$ $3.2 \mathrm{mV}$, respectively. Strikingly, when Gap19 or $\mathrm{AbCx} 43$ was added in the pipette solution, $V m$ in $D m d^{m d x}$ cardiomyocytes was returned to values similar to those observed in WT cardiomyocytes. Consistently, $D m d^{m d x} \mathrm{Cx}_{43^{+/-}}$cardiomyocytes in the absence or presence of Iso displayed resting $V m$ depolarization with $V m$ values of $-67.5 \pm 0.9 \mathrm{mV}$ and $-65.9 \pm 2.4 \mathrm{mV}$, respectively. These resting $V m$ values resemble 
A
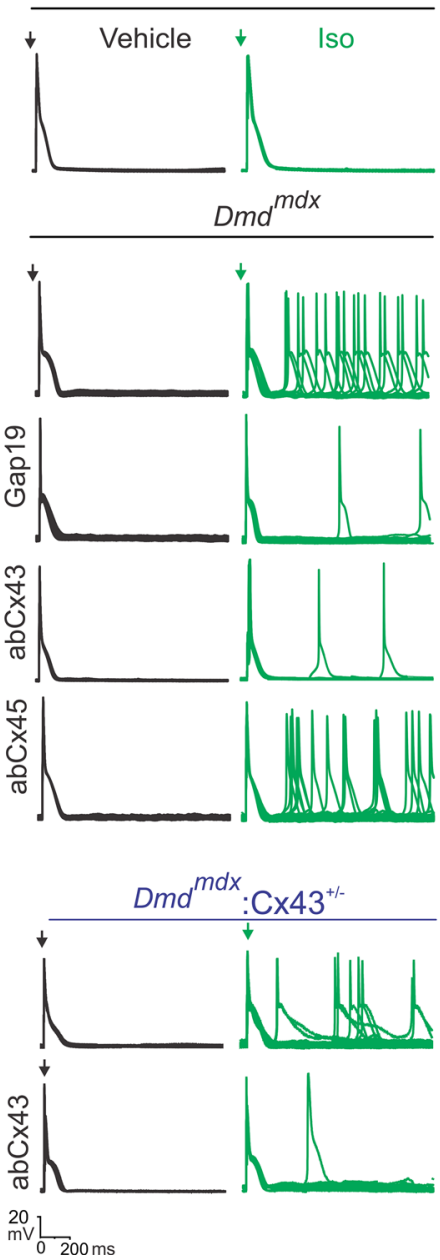

B

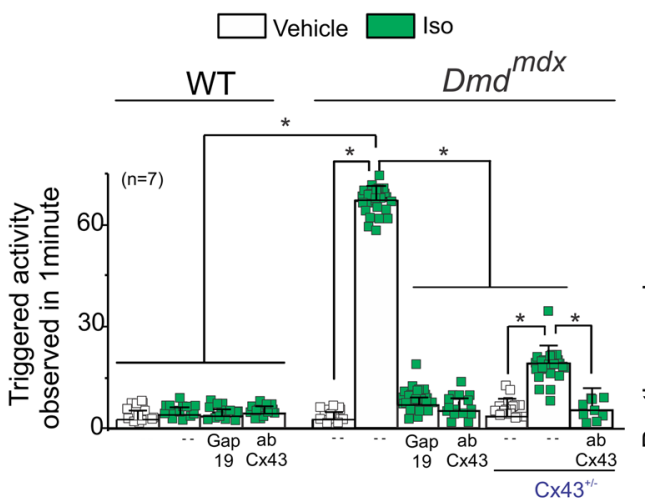

C

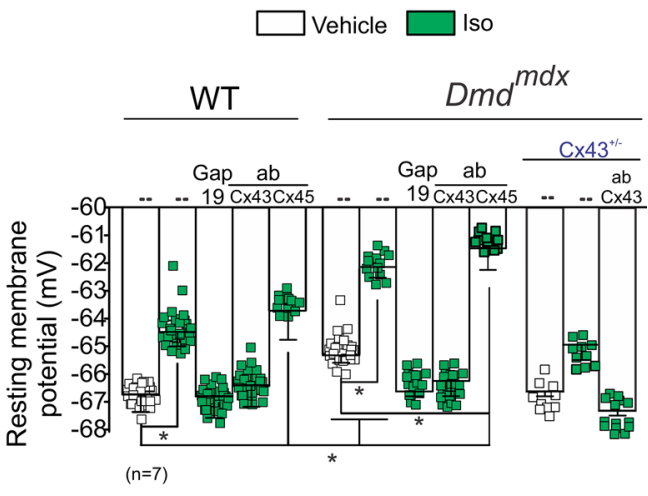

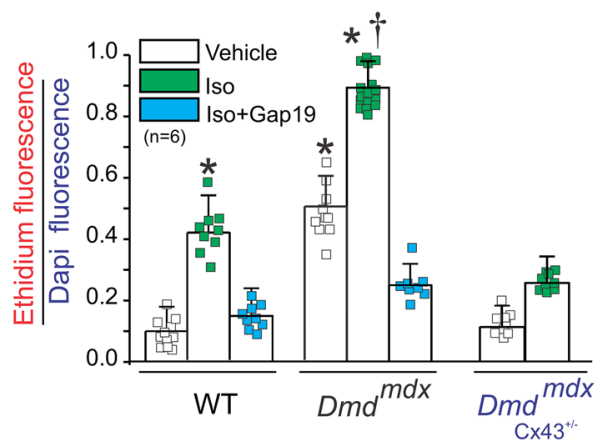

Figure 1. Isoproterenol induces triggered activity in $\mathbf{D} m \mathbf{d}^{\mathbf{m d x}}$ cardiomyocytes via opening of $\mathbf{C x} \mathbf{4 3}$ hemichannels. (A) Representative action potential (AP) traces of WT, $D m d^{m d x}$, and $D m d^{m d x} C \times 43^{+/-}$isolated cardiomyocytes. Cells were stimulated with $1 \mu \mathrm{M}$ isoproterenol (Iso, shown in green) in the absence or presence of $\mathrm{Cx} 43$ or $\mathrm{Cx} 45$ hemichannel blockers contained inside the pipette: Gap19 (232 ng/ $\mu \mathrm{L}$ ), Cx43 CT antibody (abCx43; $2.5 \mathrm{ng} / \mu \mathrm{L}$ ), or Cx45 CT antibody $(2.5 \mathrm{ng} / \mu \mathrm{L})$. Arrow indicates electrical stimulation pulse. (B) Quantification of triggered activity (TA) induced by Iso observed in $\mathbf{A}$. Comparisons between groups were made using 2-way ANOVA plus Tukey's post hoc test; ${ }^{*} P<0.05$. (C) Resting membrane potential of WT and $D m d^{m d x}$ cardiomyocytes. The number in parentheses indicates the $n$ value. Comparisons between groups were made using 2-way ANOVA plus Tukey's post hoc test; ${ }^{*} P<0.05$. (D) Assessment of Cx43 hemichannel activity in the whole heart via ethidium uptake. Isolated hearts were perfused with buffer containing $5 \mu \mathrm{M}$ ethidium after vehicle or Iso ( 5 $\mathrm{mg} / \mathrm{kg}$ IP). The number in parentheses indicates the $n$ value. Comparisons between groups were made using 2-way ANOVA plus Tukey's post hoc test. ${ }^{*} P<$ 0.05 vs. vehicle $W T^{\dagger}{ }^{\dagger} P<0.05$ vs. vehicle $D m d^{m d x}$.

more closely those observed in WT cardiomyocytes than $D m d^{m d x}$ cardiomyocytes. Intracellular application of $\mathrm{AbCx} 43$ in $D m d^{m d x} \mathrm{Cx} 43^{+/-}$cardiomyocytes further prevented isoproterenol-induced resting $\mathrm{Vm}$ depolarization to $V m$ values of $-68.2 \pm 4.9 \mathrm{mV}$. These data support that plasma membrane depolarization in $D m d^{m d x}$ cardiomyocytes is caused by the altered activity of $\mathrm{Cx} 43$ hemichannels.

To demonstrate that Iso-induced depolarization of resting $V m$ plays a crucial role in generating TA in $D m d^{m d x}$ cardiomyocytes, we injected hyperpolarizing currents to maintain the resting $V m$ near $-68 \mathrm{mV}$ (the values observed in WT cells before Iso treatment). Under these conditions, $D m d^{m d x}$ cardiomyocytes treated with Iso displayed a significant reduction of TA events (10.5 \pm 0.2 per minute) (Supplemental Figure 1; supplemental material available online with this article; https://doi.org/10.1172/ jci.insight.130091DS1). Furthermore, $D m d^{m d x}$ cardiomyocytes not treated with Iso, but injected with depolarizing currents to maintain the resting $V m$ near $-61 \mathrm{mV}$ (the value observed after Iso treatment), displayed a significant number of TAs $(60.3 \pm 0.2$ per minute).

Sodium-calcium exchanger (NCX) activity has also been proposed to promote membrane depolarization and DADs in cardiac pathologies such as hypoxia reoxygenation, transverse aortic contraction, 
and heart failure (21-24). However, blockade of NCX transporters using the specific inhibitor SEA0400 (25) did not restore Iso-induced resting $V m$ depolarization and TAs (Supplemental Figure 2) in $D m d^{m d x}$ cardiomyocytes. Taken together, our data suggest that Iso treatment produces $V m$ depolarization and, consequently, generates TA events in $D m d^{m d x}$ cardiomyocytes mainly via Cx43 hemichannel activity.

To support that Iso treatment increases activity of Cx43 hemichannels in the Dm $d^{m d x}$ hearts, we developed a semiquantitative in situ assay using perfused isolated hearts (see Methods). Uptake of hemichannel-permeable, plasma membrane-impermeable molecules, such as fluorescent ethidium bromide, from the extracellular space is used largely to measure open Cx43 hemichannels (26-28). Under normal conditions, $D m d^{m d x}$ hearts showed about 4-fold greater ethidium uptake than WT hearts (Figure 1D). Iso stimulation significantly increased ethidium uptake in both genotypes, but a significantly larger uptake was detected in $D m d^{m d x}$ hearts (Figure 1D). In vivo treatment with Gap19 via retro-orbital injection before Iso administration drastically reduced dye uptake in $D m d^{m d x}$ hearts under both normal and Iso-stimulated conditions (Figure 1D). Moreover, isolated heart from $D m d^{m d x} \mathrm{Cx}_{4} 3^{+/-}$mice did not display significant ethidium uptake upon isoproterenol treatment (Figure 1D). The uptake of a Cx43 hemichannel-impermeable dye, propidium iodide (PI), was negligible under basal or Iso-induced conditions, ruling out unspecific dye permeability mediated by plasma membrane breakdown (Supplemental Figure 3). These results indicate that the substantial ethidium uptake observed in $D m d^{m d x}$ hearts stimulated with Iso was mediated by the opening of Cx43 hemichannels.

In the next group of experiments, we estimated the amount of lateralized Cx43 hemichannels at the plasma membrane of cardiomyocytes in the intact heart of WT and Dmd ${ }^{m d x}$ mice using a modified method of cell surface protein biotinylation. Immunofluorescence against biotin perfused into whole hearts showed that biotin reached only the lateral sides of cardiomyocytes but not the intercalated discs (Figure 2A). Western blot analysis of the biotinylated fraction showed that $D m d^{m d x}$ hearts had significantly higher levels of $\mathrm{Cx} 43$ protein at the lateralized region when compared with WT hearts. Iso treatment increased the levels of lateralized Cx43 in both WT and Dmd ${ }^{m d x}$ hearts. N-cadherin and endothelial NO synthase (eNOS) were not detected in the biotinylated fraction, confirming that biotin did not interact with intercalated discs (N-cadherin) and intracellular (eNOS) proteins (Figure 2B). Furthermore, the biotinylated fraction in $D m d^{m d x} \mathrm{Cx}_{4} 3^{+/-}$hearts displayed lower levels of $\mathrm{Cx} 43$ protein at the lateralized region when compared with $D m d^{m d x}$ hearts (Supplemental Figure 4). Notably, the amount of lateralized Cx43 hemichannels at the plasma membrane strongly correlated with the levels of ethidium uptake in WT and Dmdmdx heart under control conditions and after Iso treatment (Figure 1D). Overall, our results strongly suggest an important role for Cx43 hemichannels in the pathophysiology of $D m d^{m d x}$ hearts.

Iso promotes S-nitrosylation and opening of lateralized Cx43 hemichannels in the Dmd ${ }^{m d x}$ heart. Previous reports show $\beta$-adrenergic stimulation activates NO synthases (NOSs) and promotes S-nitrosylation of several $\mathrm{Ca}^{2+}{ }_{-}$handling proteins in the heart $(29,30)$. Because opening of $\mathrm{Cx} 43$ hemichannels has been linked to NO production in astrocytes (31), we evaluated whether Cx43 is S-nitrosylated in the hearts of WT and Dmd $d^{m d x}$ mice upon Iso stimulation. Using the biotin switch assay, we found that levels of S-nitrosylated Cx43 in Dmdmdx cardiac tissue was almost 4-fold greater than in the WT heart under control conditions (Figure 3A). Iso stimulation resulted in a nearly 2-fold increase in levels of S-nitrosylated Cx43 in both WT and Dmd ${ }^{m d x}$ with respect to control (Figure 3A). S-nitrosylated Cx43 levels in $D m d^{m d x} \mathrm{Cx} 43^{+/-}$were also reduced when compared with $D m d^{m d x}$ hearts (Supplemental Figure 5A). To support that S-nitrosylation of Cx43 depends on Iso-induced NO production, both WT and $D m d^{m d x}$ animals were administered $2 \mathrm{mM} \mathrm{N}^{\omega}$-nitro-L-arginine (L-NAME), a nonselective NOS inhibitor (32), in their drinking water for 1 week before Iso stimulation. Following L-NAME treatment, levels of S-nitrosylated Cx43 in both WT and $D m d^{m d x}$ hearts treated with Iso were restored to control levels (Figure 3A).

To examine the subcellular localization of S-nitrosylated $\mathrm{Cx} 43$, we performed the proximity ligation assay (PLA) using antibodies that recognize S-nitrosylated proteins and the C-terminus of Cx43. In WT hearts, S-nitrosylated Cx43 was confined to intercalated discs, and its fluorescence was intensified after mice were injected with Iso $(5 \mathrm{mg} / \mathrm{kg})$. In $D m d^{m d x}$ hearts under control conditions, the staining for S-nitrosylated $\mathrm{Cx} 43$ was also localized to intercalated discs and was visibly stronger than WT. After treatment with Iso, S-nitrosylated Cx43 was found mostly at lateral sides in $D m d^{m d x}$ hearts. Importantly, the S-nitrosylated Cx43 signals were visibly diminished in Iso-treated WT and $D m d^{m d x}$ hearts after 1 week of L-NAME administration in the drinking water (Figure 3B). $D m d^{m d x} \mathrm{Cx} 43^{+/-}$cardiomyocytes also displayed a significant reduction of S-nitrosylated Cx43 signals compared with $D m d^{m d x}$ and WT hearts in the absence or presence of $\beta$-adrenergic stress (Supplemental Figure 5B). These data show that S-nitrosylated Cx 43 was localized to the lateral side of $D m d^{m d x}$ cardiomyocytes upon Iso treatment. 
A
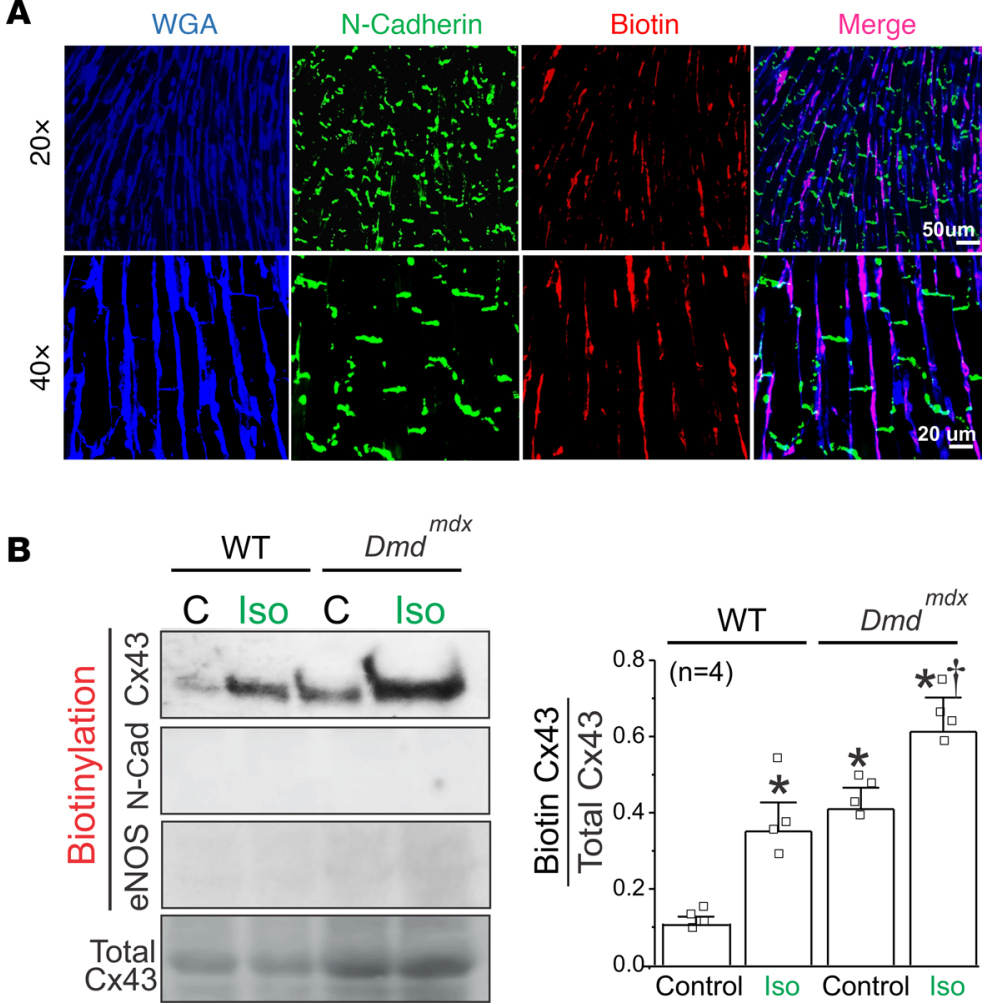

Figure 2. Biotin perfused in intact hearts interacts only with plasma membrane of cardiomyocytes at the lateral side. (A) Representative immunofluorescence against $\mathrm{N}$-cadherin (green) and biotin (red) in a $D m d^{m d x}$ heart sample. Biotin was perfused for 60 minutes before heart fixation. Cryosections were stained with wheat germ agglutinin (WCA, blue). Note that biotin was positively stained only at the lateral borders of cardiomyocytes and not at intercalated discs. (B) Western blot analysis and quantification of $[x 43$ from biotin-perfused hearts (biotinylation). Bottom row represents $[x 43$-immunoblotted samples from heart lysates prior to pulldown (total Cx43). Biotinylated Cx43 levels were expressed as fold change relative to total $\mathrm{Cx} 43$ protein levels per sample. Note that biotin did not interact with intracellular proteins (eNOS) and intercalated disc proteins (N-cadherin). The number in parentheses indicates the $n$ value. Comparisons between groups were made using 2-way ANOVA plus Tukey's post hoc test. ${ }^{*} P<0.05$ vs. WT control; ${ }^{\dagger} P<0.05$ vs. $D m d^{m d x}$ control.

To evaluate whether Iso-induced NO production and S-nitrosylation of Cx43 are linked to an increase in Cx43 hemichannel activity, we examined whether Cx43 hemichannel-mediated ethidium uptake in perfused WT and $D m d^{m d x}$ hearts is inhibited by pretreatment with L-NAME. Ethidium fluorescence increased by about 2-fold with respect to vehicle conditions in the $D m d^{m d x}$ heart after Iso treatment (Figure 3C). Moreover, L-NAME treatment completely eliminated Iso-induced ethidium uptake. These data suggest that $\beta$-adrenergic stimulation-induced ethidium uptake in $D m d^{m d x}$ mice results from the opening of S-nitrosylated Cx43 hemichannels.

We also performed biotin switch assay and PLA analysis on heart samples obtained from multiple non-DMD and DMD patients to test S-nitrosylation status of Cx43 in humans. Total S-nitrosylated levels of $\mathrm{Cx} 43$ in the human DMD hearts were 3-fold higher than those observed in controls (Figure 3D). PLA analysis showed that S-nitrosylated Cx43 was located at the lateral sides of human DMD hearts. Conversely, in non-DMD hearts, S-nitrosylated Cx43 was located mainly at the intercalated discs (Figure $3 \mathrm{E}$ ). These findings indicate that S-nitrosylated Cx43 might play an essential role in human DMD cardiomyopathy.

Inhibition of NOS prevents Iso-induced TAs and arrhythmias in Dmdmdx mice. Because Iso-evoked opening of Cx43 hemichannels was drastically reduced by inhibiting NO production in $D m d^{m d x}$ hearts (Figure 3C), we next studied whether TAs mediated by $\mathrm{Cx} 43$ hemichannels were also affected by inhibition of NO production in Iso-treated $D m d^{m d x}$ cardiomyocytes. Figure 4A shows representative APs in WT and Dmdmdx isolated cardiomyocytes that were evoked by electrical stimulation in control conditions or in the presence of 100 $\mu \mathrm{M}$ L-NAME. Treatment with L-NAME reduced the incidence of Iso-evoked TAs in Dmd ${ }^{m d x}$ cardiomyocytes (Figure 4, A and B). Moreover, L-NAME significantly reduced the Iso-induced increase in the resting 
A

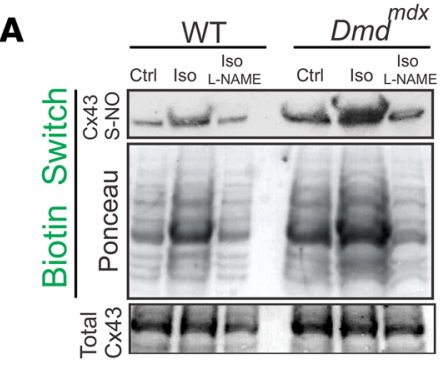

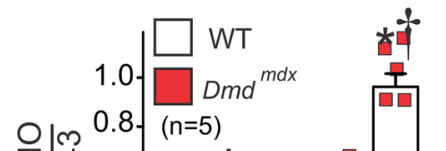

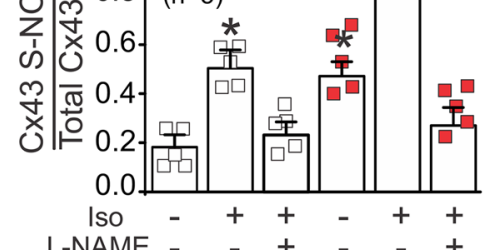

C

\begin{tabular}{c|cc|c}
\multicolumn{4}{c}{$D m d^{m d x}$} \\
\hline Vehicle & \multicolumn{1}{c}{ Iso } & L-NAME \\
\hline $\begin{array}{c}\text { L-No } \\
\searrow\end{array}$ & $\searrow$ & $\searrow$ & $\searrow$ \\
$\searrow$ & $\searrow$ & $\searrow_{10 \text { um }}$
\end{tabular}

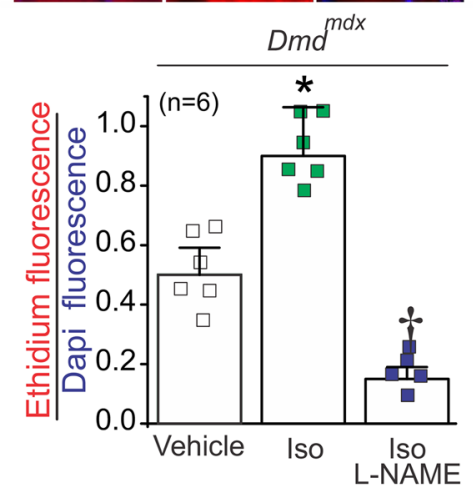

D
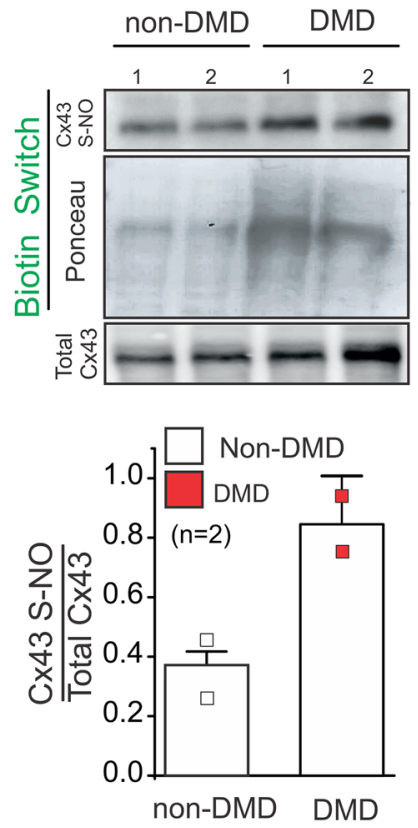

B
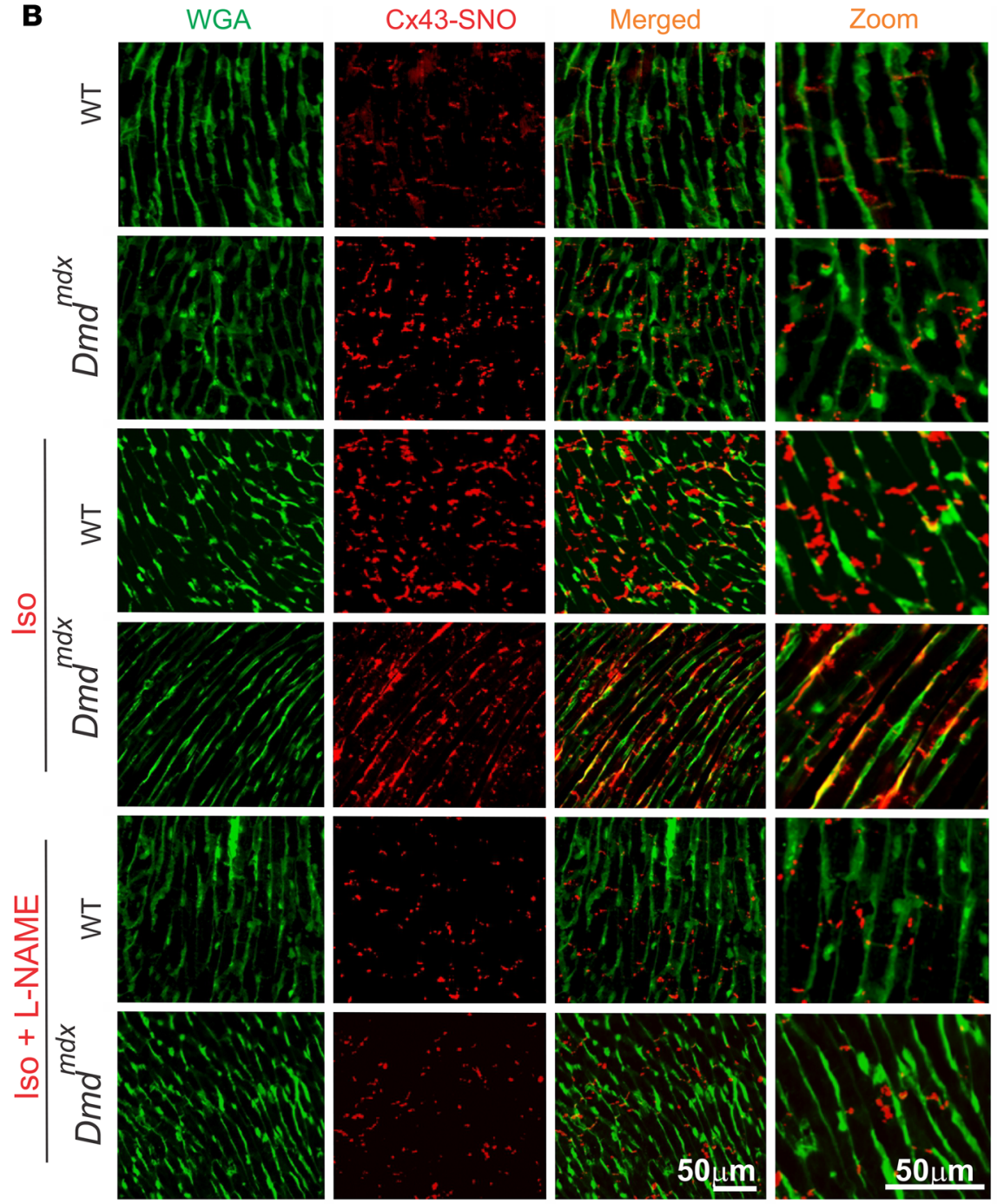

E

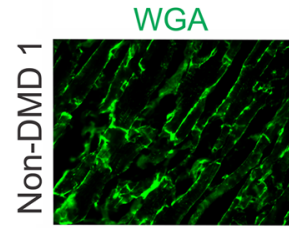

CX43-SNO

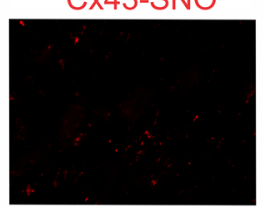

Merged
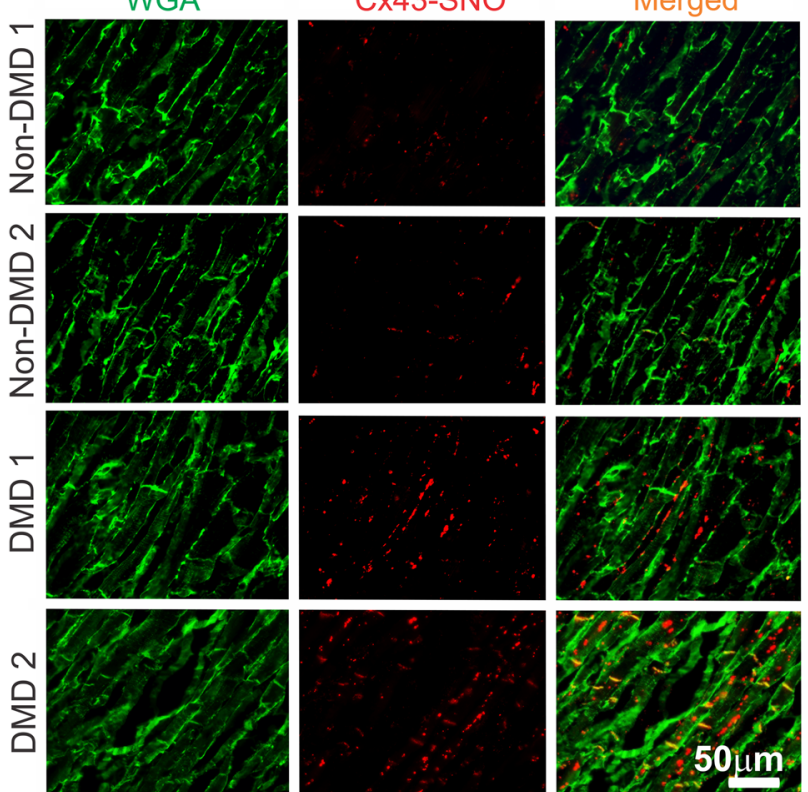

Zoom
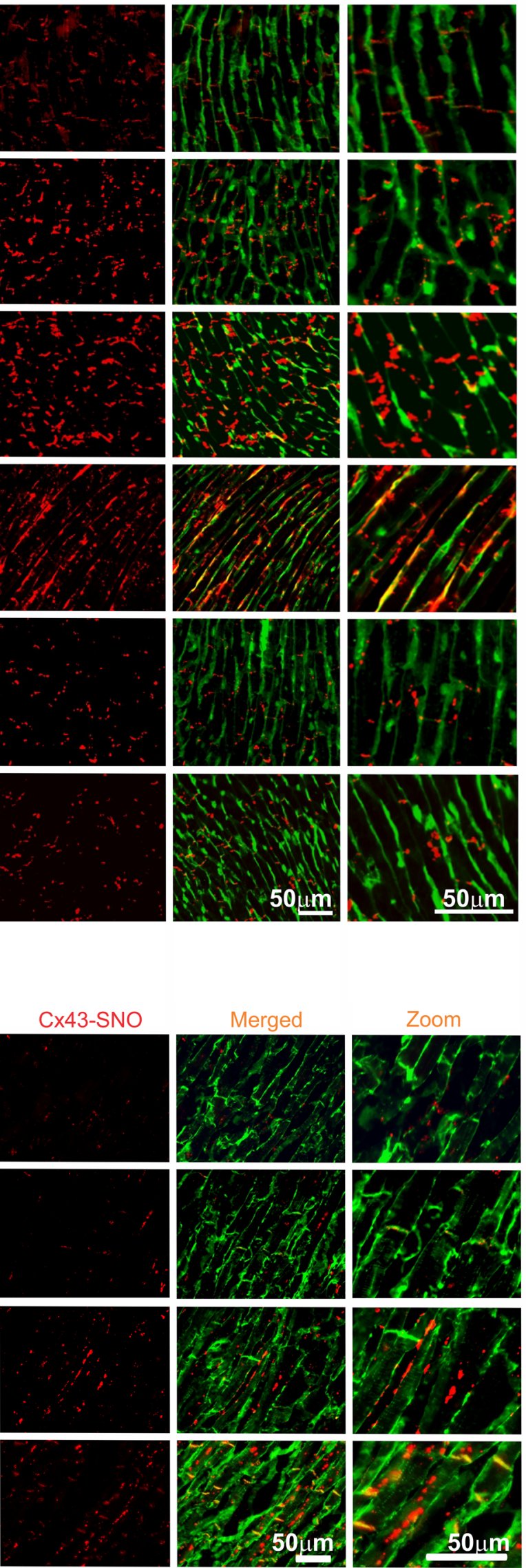

$50 \mathrm{im}$
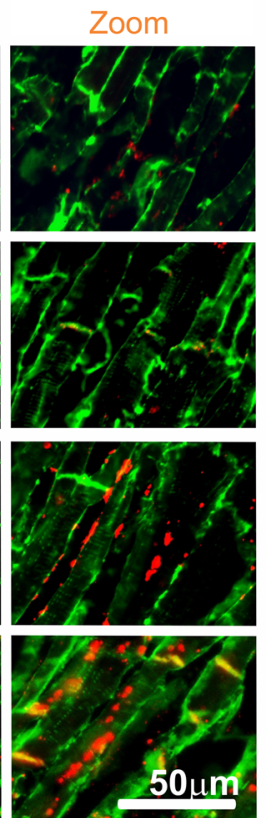
Figure 3. Iso increases S-nitrosylated levels of Cx43 at the lateral side of $\mathbf{D m}^{\mathrm{mdx}}$ cardiomyocytes. (A) Top and middle gels were loaded with S-nitrosylated proteins pulled down from heart samples using the biotin switch assay. Top gel was, then, blotted against Cx43, and the middle gel is the corresponding Ponceau staining. Lower blot was loaded using total cardiac proteins and blotted against Cx43. The bottom graph is the quantification for 5 independent blots using the ratio for S-nitrosylated Cx43/Ponceau. The number in parentheses indicates the $n$ value. Comparisons between groups were made using 2-way ANOVA plus Tukey's post hoc test. ${ }^{*} P<0.05$ vs. WT control, and ${ }^{\dagger} P<0.05$ vs. WT Iso. (B) Analysis performed by PLA of the interaction between $\mathrm{Cx} 43$ and S-nitrosylation. Plasma membrane stained with wheat germ agglutinin (WCA) and S-nitrosylated Cx43 (Cx43SNO) are shown in green and red, respectively. Representative images of $n=5$ per group. (C) Assessment of Cx43 hemichannel activity in isolated $D m d^{m d x}$ hearts perfused with buffer containing $5 \mu \mathrm{M}$ ethidium bromide after or without treatment with Iso. Arrows show nuclei. The number in parentheses indicates the $n$ value. Comparisons between groups were made using 2 -way ANOVA test plus Tukey's post hoc test. ${ }^{*} P<0.05$ vs. vehicle $\mathrm{WT}^{\dagger}{ }^{\dagger} P<0.05$ vs. vehicle $D m d^{m d x}$. (D) Top and middle gels were loaded with S-nitrosylated proteins pulled down from human heart samples using the biotin switch assay. Top gel was, then, blot against $\mathrm{C} \times 43$ and the middle gel is the corresponding ponceau staining. Lower blot was load using total cardiac proteins and blot against Cx43. (E) Analysis performed by PLA of the interaction between Cx43 and S-nitrosylation in human samples. Note that $\mathrm{Cx} 43$ is S-nitrosylated at the lateral side of DMD human samples compare to non-DMD.

$V m$ of $D m d^{m d x}$ cardiomyocytes, restoring it to similar values observed in WT cardiomyocytes (Figure 4C). L-NAME's effect on TAs was similar to that observed after the block of Cx43 hemichannels (Figure 1, A and C), supporting the idea that $\mathrm{Cx} 43$ hemichannel-mediated aberrant electrical activity in Dmd ${ }^{m d x}$ cardiomyocytes is likely the result of S-nitrosylation of hemichannels.

Because we have proposed that Cx43 hemichannels mediate Iso-induced arrhythmogenesis in $D m d^{m d x}$ mice $(16,18)$, we next tested whether blockade of NO production reduces arrhythmias. To test this, we performed in vivo electrocardiograms (ECGs) in WT and $D m d^{m d x}$ mice under control condition and after L-NAME treatment. Representative ECGs recorded from WT mice and $D m d^{m d x}$ mice treated with L-NAME are shown in Figure 4D. Remarkably, $D m d^{m d x}$ mice treated with L-NAME were protected from Iso-induced arrhythmias (Figure 4D), which was evident by a significantly lower arrhythmia score compared with vehicle-treated counterparts (Figure 4E).

Exogenous application of NO induces TA in Dmd ${ }^{m d x}$ cardiomyocytes. To demonstrate a direct role of NO on the generation of TA in $D m d^{m d x}$ cardiomyocytes, we next investigated whether application of an NO donor, sodium 2-(N,N-diethylamino)-diazenolate-2-oxide (DEENO, $1 \mu \mathrm{M})$, mimics Iso-induced TA. TAs were observed in DEENO-treated $D m d^{m d x}$ isolated cardiomyocytes $(58.1 \pm 4.3$ per minute) but not WT (Figure 5, A and B). This value is comparable to that observed for Iso-induced TA (Figure 1C). Furthermore, $\mathrm{Cx} 43$ hemichannel blockers, Gap19 and $\mathrm{AbCx} 43$, largely reduced observed TAs to values of $8.2 \pm 0.9$ and $7.3 \pm 0.8$, respectively (Figure $5, \mathrm{~A}$ and $\mathrm{B}$ ). As expected, isolated cardiac cells from $D m d^{m d x} \mathrm{Cx} 43^{+/-}$mice displayed a significant reduction of TAs (10.4 \pm 1.2 per minute) upon DEENO stimulation when compared with $D m d^{m d x}$ cardiomyocytes (Figure 5, A and B). In addition, exogenous NO application depolarized the membrane in both WT and $D m d^{m d x}$ cardiomyocytes to membrane potentials of -65.9 and $-61.8 \mathrm{mV}$, respectively (Figure 5C). Cx43 hemichannel blockers and genetic reduction of the levels of $\mathrm{Cx} 43\left(D m d^{m d x} \mathrm{Cx}_{4} 3^{+/-}\right.$) restored the resting membrane potentials to those observed in vehicle values (Figure 5C). Importantly, NO-treated $D m d^{m d x}$ cardiomyocytes displayed resting $V m$ values similar to those found in Iso-treated cells, thus indicating a similar mechanism of changes in membrane excitability (Figure 1C). Thus, our findings strongly suggest that Iso, NO, and Cx43 hemichannel openings operate in the same signaling pathway that induces TA and arrhythmias in stressed Dmd ${ }^{m d x}$ hearts.

NO activates CX43 hemichannels via S-nitrosylation of cysteine 271. To confirm that NO activates Cx43 hemichannels via S-nitrosylation, we assessed Cx43 hemichannel ionic currents in Xenopus oocytes using the 2-electrode voltage clamp technique. Consistent with previous studies, we did not observe noticeable $\mathrm{Cx} 43$ hemichannel currents in response to changes in voltage or low extracellular $\left[\mathrm{Ca}^{2+}\right](26,33-36)$. However, treatment of Cx43-expressing oocytes with $10 \mu \mathrm{M}$ DEENO evoked an increase in plasma membrane conductance at all voltages when compared with noninjected oocytes (Figure 6A). The increase in current induced by NO was blocked when using either Gap19 or AbCx43 (Figure 6B), showing that observed current originated from $\mathrm{Cx} 43$ hemichannels.

We also found NO induced depolarization of the $V m$ in oocytes expressing Cx43 but not in the noninjected oocytes. Consistently, Gap19 and AbCx43 prevented NO-induced Vm depolarization in Cx43-expressing oocytes (Figure 6C). Extracellular calcium drastically reduces $\mathrm{Cx} 43$ open hemichannel probability (26). Consistent with this, NO-induced $V m$ changes in $\mathrm{Cx} 43$-expressing oocytes were dependent on extracellular calcium concentrations (Figure 6D).

The C-terminus of $\mathrm{Cx} 43$ is a target for various posttranslational modifications. It includes various phosphorylation sites that affect gap junction plaque formation and stability (37-39). It also contains 
A

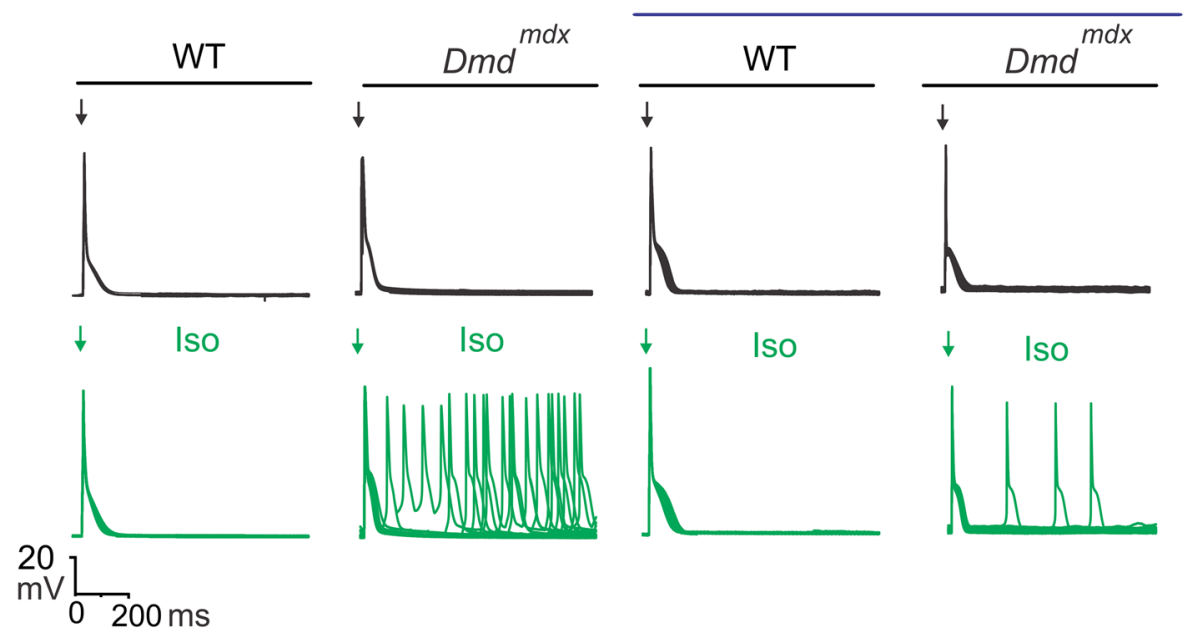

B

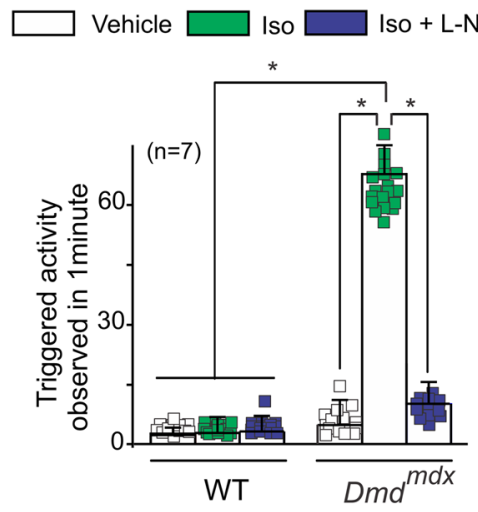

D

Baseline

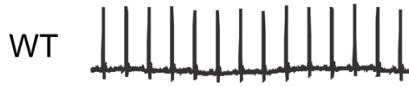

WT

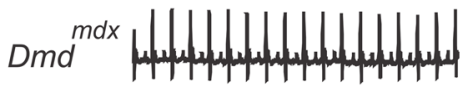

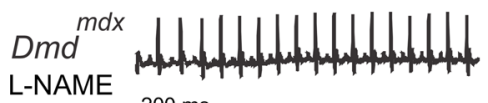

$200 \mathrm{~ms}$

\section{C}

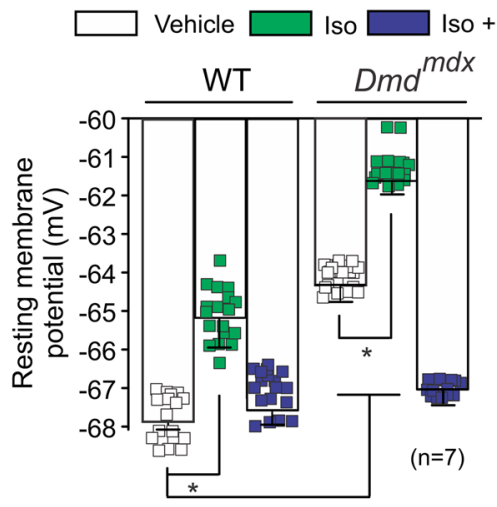

Iso

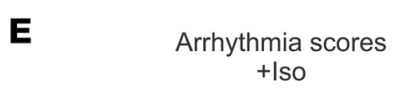

E

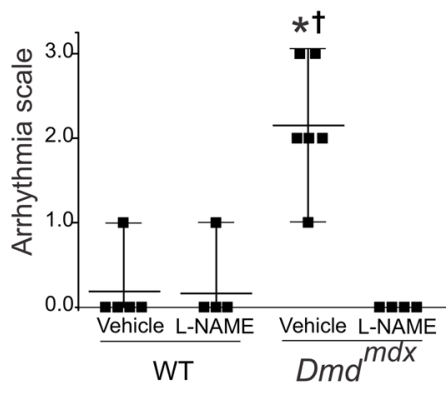

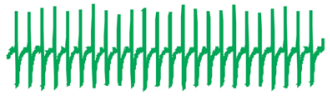
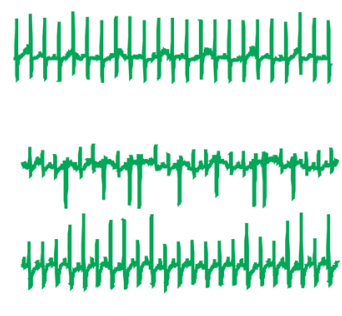

Figure 4. Blockade of NO production prevents $\mathrm{Cx} 43$ hemichannelmediated TA and arrhythmias in Dmd $^{\text {ddx }}$ mice. (A) Representative action potential traces of WT and $D m d^{m d x}$ isolated cardiomyocytes. Cells were stimulated with $1 \mu \mathrm{M}$ Iso in the presence of $100 \mu \mathrm{M}$ L-NAME. Arrow indicates electrical stimulation. (B) Quantification of TA induced by Iso observed in $\mathbf{A}$. The number in parentheses indicates the $n$ value. Comparisons between groups were made using 2-way ANOVA plus Tukey's post hoc test; ${ }^{*} P<0.05$. (C) Resting membrane potential of WT and Dmd ${ }^{m d x}$ cardiomyocytes. Comparisons between groups were made using 2-way ANOVA plus Tukey's post hoc test. (D) Representative ECG traces of 5- to 6-month-old WT and Dmd ${ }^{m d x}$ mice that were previously treated or not with $2 \mathrm{mM}$ L-NAME (an unspecific NOS blocker) via drinking water. ECG baseline and ECG after Iso treatment $(5 \mathrm{mg} / \mathrm{kg} \mathrm{IP})$ are shown for comparison. (E) Arrhythmia score based on predetermined scale: 0 , no arrhythmias; 1 , single premature ventricular contractions (PVCs); 2, double PVCs; 3, triple PVCs or nonsustained ventricular tachycardia (VT); 4, sustained VT or atrioventricular (AV) block; and 5 , death. ${ }^{*} P<0.0001$ versus $W T ;{ }^{\dagger} P<$ 0.0001 versus $D m d^{m d x}$ L-NAME. The number in parentheses indicates the $n$ value. Statistical significance determined by 1-way ANOVA plus Tukey's post hoc test.

3 cysteines (C260, C271, and C298) that could be targets of S-nitrosylation. Thereby, we deleted the C-terminus of $\mathrm{Cx} 43(\mathrm{CT}, \mathrm{Cx} 43 \Delta \mathrm{CT})$ and examined ionic currents in response to DEENO application. Cx43 $\triangle$ CT-expressing oocytes did not display NO-induced hemichannel currents upon stimulation with DEENO (Figure 7A). We also made single substitutions of cysteines C260S, C271S, and C298S of Cx43 with nonpolar amino acid serine and tested for NO-induced hemichannel activation. Whereas C260Sand C298S-mutant hemichannels remained sensitive to NO, the C271S mutant completely lost its NO dependence (Figure 7A). Moreover, DEENO treatment depolarized the resting $V m$ in oocytes expressing C260S and C298S but not C271S (Figure 7B).

Although the above data suggest that C271 is critical for NO-induced Cx43 hemichannel opening, we cannot rule out yet the possibility that the deletion of the $\mathrm{CT}$ in $\mathrm{Cx} 43$ and the $\mathrm{C} 271 \mathrm{~S}$ mutation per se preclude 
A NO

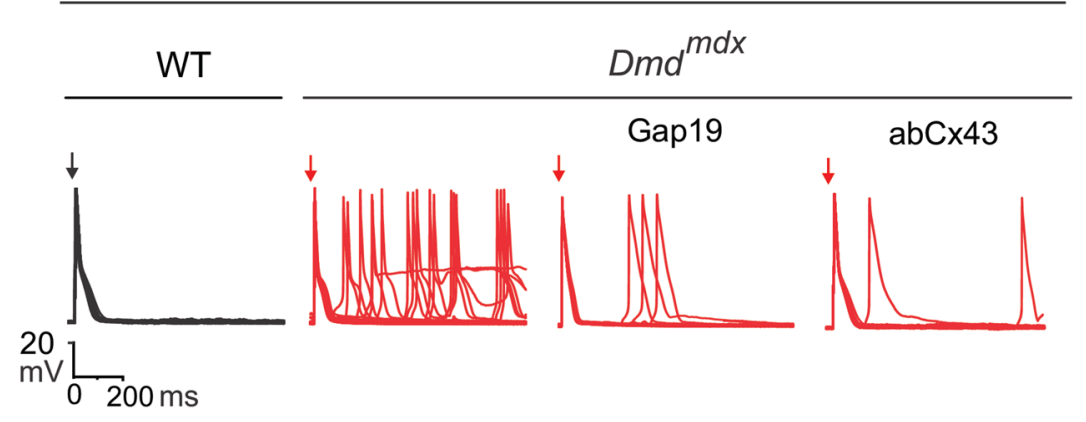

$D m d^{m d x}: \mathrm{C} \times 43^{+/}$

B

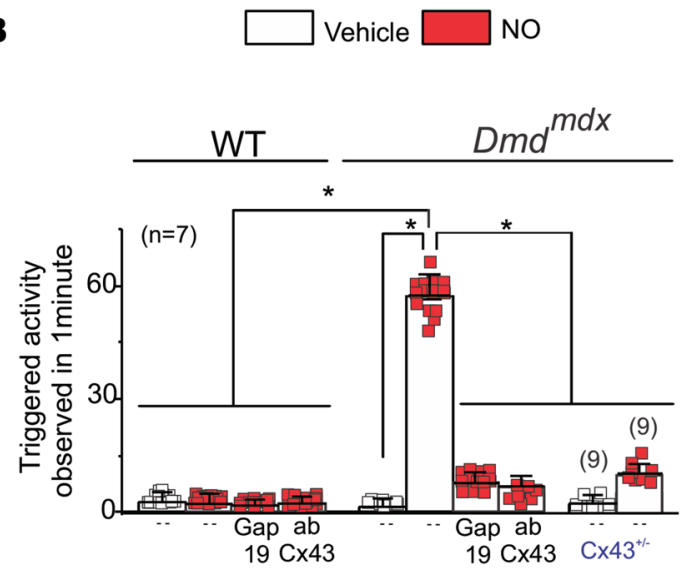

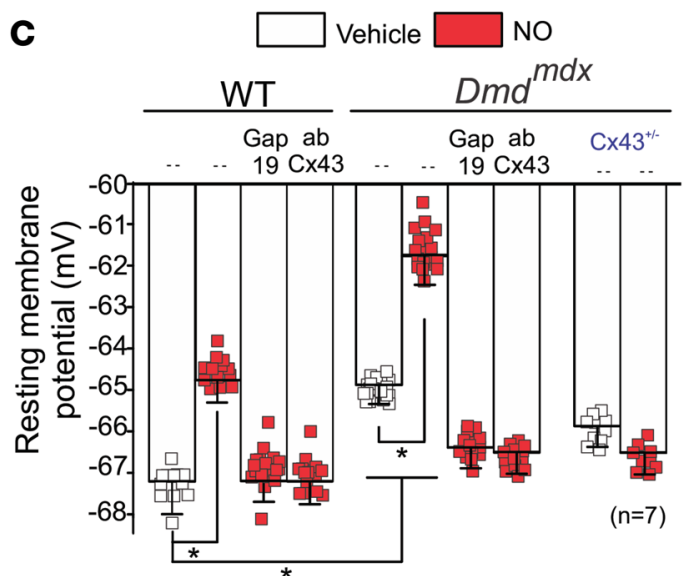

Figure 5. Exogenous NO-induced TA in Dmd ${ }^{m d x}$ cardiomyocytes. (A) Representative AP traces of WT, $D m d^{m d x}$, and $D m d^{m d x} C \times 43^{+/-}$isolated cardiomyocytes. Cells were stimulated with $1 \mu \mathrm{M}$ DEENO in the absence or presence of Cx43 hemichannel blockers, Gap19 (232 ng/ $\mu \mathrm{L}$ ) and Cx43 CT antibody (abCx43; 2.5 $\mathrm{ng} / \mu \mathrm{L}$ ). Arrow indicates electrical stimulation pulse. (B) Quantification of TA induced by DEENO in $\mathbf{A}$. The number in parentheses indicates the $n$ value. Comparisons between groups were made using 2-way ANOVA plus Tukey's post hoc test; ${ }^{*} P<0.05$. (C) Resting membrane potential of WT and $D m d^{m d x}$ cardiomyocytes upon DEENO stimulation. Comparisons between groups were made using 2-way ANOVA plus Tukey's post hoc test; ${ }^{*} P<0.05$.

hemichannel opening by affecting gating, independently of lack of nitrosylation at C271. To address this, we tested the NO responses in heteromeric $\mathrm{Cx} 43$ and $\mathrm{Cx} 26 \mathrm{~S} 17 \mathrm{~F}$ hemichannels, which are activated by changes in $V m$ (33). Supplemental Figure 6A shows representative currents from heteromeric hemichannels formed by combination of $\mathrm{Cx} 43, \mathrm{Cx} 43 \Delta \mathrm{CT}$, or $\mathrm{Cx} 43 \mathrm{C} 271 \mathrm{~S}$ and $\mathrm{Cx} 26 \mathrm{~S} 17 \mathrm{~F}$ (black traces) elicited by depolarizing pulses from $-80 \mathrm{mV}$ to $0 \mathrm{mV}$. In the presence of $10 \mu \mathrm{M}$ DEENO, heteromeric channels containing the full-length $\mathrm{Cx} 43$, but not $\mathrm{Cx} 43 \mathrm{C} 271 \mathrm{~S}$ and $\mathrm{Cx} 43 \Delta \mathrm{CT}$, displayed an increase in currents (red traces). $\mathrm{Cx} 26$ or $\mathrm{Cx} 26 \mathrm{~S} 17 \mathrm{~F}$ homomeric hemichannels were not sensitive to NO donors (Supplemental Figure 6B). Thus, we conclude that the CT of $\mathrm{Cx} 43$ mediates NO-induced hemichannel currents via NO modifications at the residue C271.

To biochemically confirm that residue C271 is S-nitrosylated by NO in Cx43 hemichannels, we conducted a biotin switch assay. S-nitrosylated Cx43 was detected in oocytes expressing Cx43, Cx43C260S, and Cx43C298S after the treatment with $10 \mu \mathrm{M}$ DEENO. Consistent with electrophysiological recordings, Cx43C271S did not display detectable levels of S-nitrosylation after treatment with DEENO (Figure 7C). These results confirm that $\mathrm{C} 271$ is the critical residue, which is the subject of modification by NO, and is expected to participate in $\mathrm{Cx} 43$ hemichannel opening in response to S-nitrosylation in vivo.

\section{Discussion}

We previously reported overexpression and pathological remodeling of Cx43 in the hearts of DMD patients and $D m d^{m d x}$ mice (16). $\beta$-Adrenergic stimulation with Iso caused severe arrhythmias and sudden death in $D m d^{m d x}$ mice, which were prevented by either administration of $\mathrm{Cx} 43$ hemichannel blockers or genetically reducing Cx43 protein levels $(16,18)$. In the present study, we examined cellular and molecular mechanisms by which $\mathrm{Cx} 43$ proteins mediate cardiac stress-evoked arrhythmias. We established that $\beta$-adrenergic stimulation with Iso promotes changes in membrane permeability and TAs in $D m d^{m d x}$ cardiomyocytes via opening of lateralized and S-nitrosylated Cx43 hemichannels. Normalization of the S-nitrosylation-redox balance by 
A
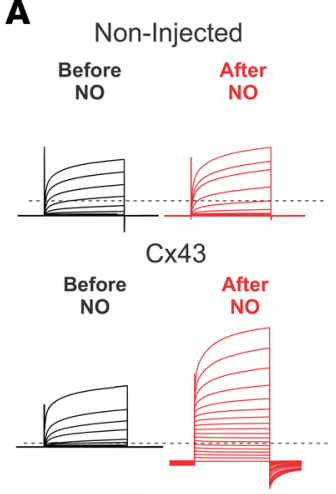

$\left.0.5 \mu \mathrm{A}\right|_{1 \mathrm{~s}}$

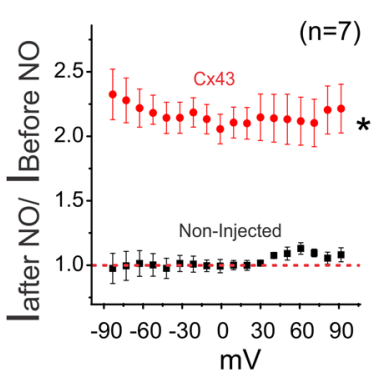

C

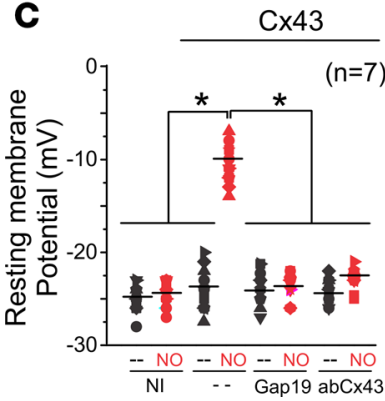

B
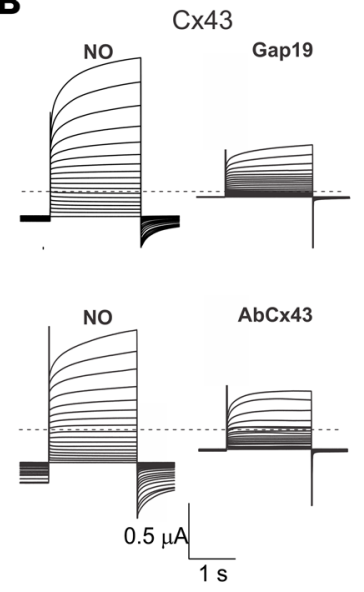

$\square$ Cx43 with Gap19

Cx43 with $\mathrm{AbCx43}$

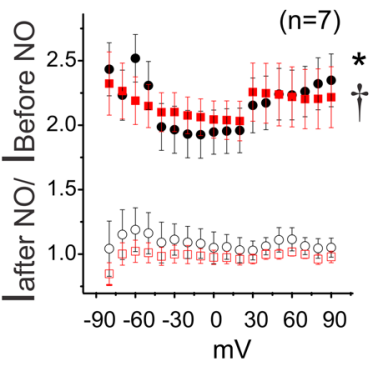

D

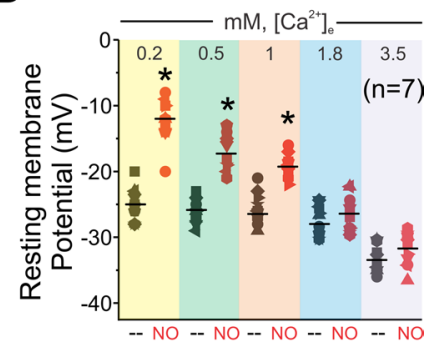

Figure 6. NO-activated currents from oocytes expressing $\mathrm{Cx} 43$ hemichannels. (A) Representative current traces before and after application of $10 \mu \mathrm{M}$ DEENO in a noninjected oocyte or an oocyte expressing $C x 43$. Oocytes were clamped to $-80 \mathrm{mV}$, and square pulses from -80 $\mathrm{mV}$ to $+90 \mathrm{mV}$ (in 10-mV steps) were then applied for 2 seconds. At the end of each pulse, the membrane potential was returned to $-80 \mathrm{mV}$. Normalized currents were obtained from the ratio between recorded current after and before DEENO treatment. The number in parentheses indicates the $n$ value. Comparisons between groups were made using 2-way ANOVA plus Tukey's post hoc test; ${ }^{*} P<0.05$ vs. noninjected. (B) Intracellular injection of Cap19 (232 $\mathrm{ng} / \mu \mathrm{L}$ ) or a $\mathrm{Cx} 43 \mathrm{CT}$ antibody $(2.5 \mathrm{ng} / \mu \mathrm{L})$ reduced $\mathrm{NO}$-induced $\mathrm{C} \times 43$ hemichannel currents. The number in parentheses indicates the $n$ value. Comparisons between groups were made using 2-way ANOVA plus Tukey's post hoc test. ${ }^{*} P$ $<0.05$ vs. Cx43 with Gap19; ${ }^{\dagger} P<0.05$ vs. $C \times 43$ with $A b C \times 43$. (C) Changes in resting membrane potential in the presence or absence of $10 \mu \mathrm{M}$ DEENO. Cx43 hemichannel blockers restored normal resting membrane potential. Comparisons between groups were made using 1-way ANOVA; ${ }^{*} P<0.05$. (D) Extracellular calcium dependence of the resting membrane potential evoked by $10 \mu \mathrm{M}$ DEENO in oocytes expressing CX43 hemichannels. The number in parentheses indicates the $n$ value. Comparisons between groups were made using 2-tailed Student's $t$ test; ${ }^{*} P<0.05$ vs. control.

inhibition of the NOSs reversed changes in membrane permeability, halted TAs, and prevented arrhythmogenic behavior in the $D m d^{m d x}$ mice. Finally, we demonstrated that S-nitrosylation at the residue C271 promotes opening of Cx43 hemichannels. This is a strong indication of a critical role of S-nitrosylated lateralized Cx43 hemichannels in the developing of arrhythmias in $D m d^{m d x}$ mice.

Over time, $\beta$-adrenergic-induced arrhythmias in $D m d^{m d x}$ mice progress from premature ventricular contractions to ventricular tachycardia and AV block $(16,18)$. These arrhythmic events did not arise as an autonomic defect, such as parasympathetic surge, because they were also observed in isolated $D m d^{m d x}$ hearts perfused with Iso (18). Our data suggest that $\beta$-adrenergic-induced ventricular arrhythmias were evoked primarily by TA and increased activity of Cx43 hemichannels. It is well known that TA can culminate in sustained abnormal heart rhythms due to early and delayed after-depolarizations, which are associated with $V m$ oscillations after the upstroke of an AP (40-42). $\beta$-Adrenergic stimulation depolarizes the plasma membrane in both WT and $D m d^{m d x}$ cardiomyocytes (10). However, membrane depolarization in $D m d^{m d x}$ cardiomyocytes was significantly greater and sufficient to promote TAs. Application of Cx43 hemichannel blockers restored the resting $V m$ in Iso-treated $D m d^{m d x}$ cardiomyocytes to WT levels and markedly decreased TAs. Our data rule out the role of NCX transporters, which has been implicated in the generation of DADs and arrhythmias in other cardiac pathologies (22-24). This result is also in line with previous studies indicating that $\beta$-adrenergic stimulation did not activate NCX current under normal conditions 
A

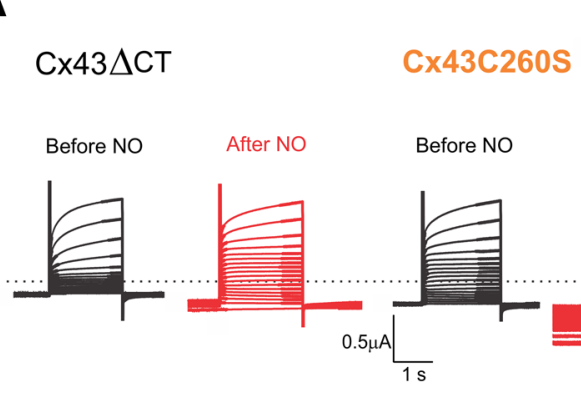

After NO

Cx43C271S

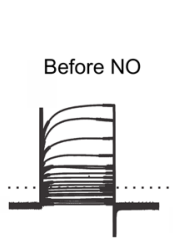

\section{(1)}

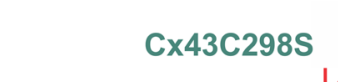

After NO
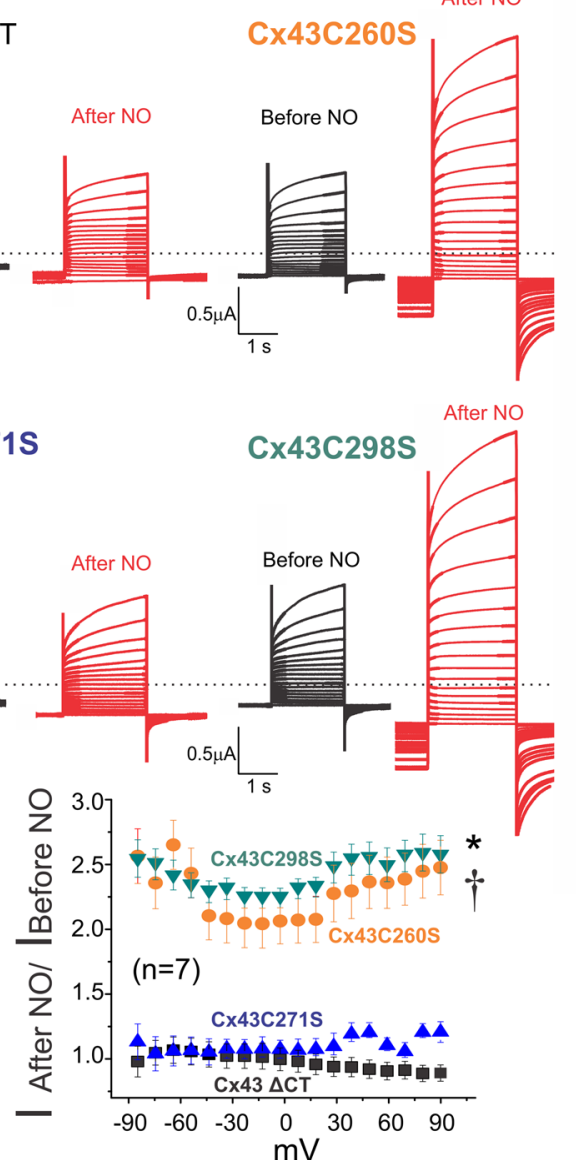

B
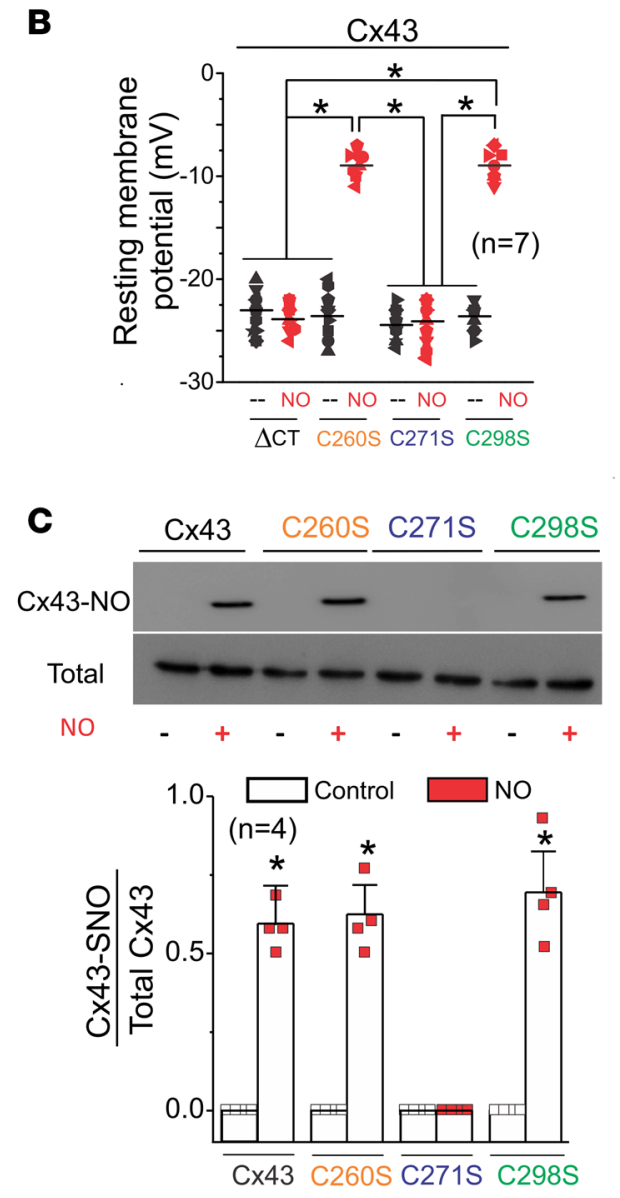

Figure 7. Position C271, but not C260 and C298, was S-nitrosylated and mediated NO-induced hemichannel currents. (A) Representative current traces for oocytes expressing Cx43 with a deleted CT and Cx43 mutants C260S, C271S, and C2985. Black and red traces correspond to voltage step-evoked currents in the absence or presence of $10 \mu \mathrm{M}$ DEENO, respectively. Oocytes were clamped to $-80 \mathrm{mV}$, and square pulses from $-80 \mathrm{mV}$ to $+90 \mathrm{mV}$ (in 10 -mV steps) were then applied for 2 seconds. At the end of each pulse, the membrane potential was returned to -80 mV. Graph shows normalized fold increase of DEENO current after treatment at different voltages. The number in parentheses indicates the $n$ value. Comparisons between groups were made using 2 -way ANOVA plus Tukey's post hoc test; ${ }^{*} P<0.05$ vs. Cx43 $\triangle \mathrm{CT} ;{ }^{\dagger} P<0.05 \mathrm{vs}$. Cx43C271. (B) DEENO decreased the resting membrane potential in oocytes expressing Cx43 mutants $C 2605$ and $C 2985$ but not in those expressing the $C x 43 \Delta C T$ or $C x 43$ mutant $C 2715$. The number in parentheses indicates the $n$ value. Comparisons between groups were made using 2-way ANOVA test; ${ }^{*} P<0.05$ vs. control. (C) Top gel was loaded with S-nitrosylated proteins pulled down using the biotin switch assay and blotted against $C \times 43$. Bottom Western blot was loaded with total proteins of oocytes expressing $C \times 43$ against Cx43. The number in parentheses indicates the $n$ value. Comparisons between groups were made using Student's $t$ test; ${ }^{*} P<0.05$ vs. control.

in guinea pig, mouse, and rat ventricular myocytes (43). In addition, $\mathrm{K}^{+}$currents, which maintained and restored resting $V m$ following AP generation, were not affected by the Cx43 hemichannel blocker Gap19 in both WT and $D m d^{m d x}$ cardiomyocytes (Supplemental Figure 7).

Our above data indicate that Iso depolarizes $V m$ and produces TAs mainly via $\mathrm{Cx} 43$ hemichannel activity. This idea is further supported by measurements of ethidium uptake across the plasma membrane of intact hearts. Iso treatment substantially increased membrane permeability in $D m d^{m d x}$ but not WT hearts, which nicely correlates with our findings of significant changes in the Vm and the presence of TAs in isolated $D m d^{m d x}$ but not WT cardiomyocytes. Hearts from 4- to 5-month-old $D m d^{m d x}$ mice displayed abnormal membrane permeability, but cardiac dysfunction was undetectable unless mice were subjected to cardiac stress. Because cardiac pathology does not emerge until $D m d^{m d x}$ mice are at least 8 months old, the increase in the membrane permeability might serve as an early sign of DMD cardiomyopathy.

The high conductance and poor selectivity of $\mathrm{Cx} 43$ hemichannels (26) combined with increased levels of lateralized Cx43 hemichannels in $D m d^{m d x}$ cardiomyocytes should cause severe membrane depolarizations if $\mathrm{Cx} 43$ hemichannels are fully open. However, we observed only a rightward shift of 6-8 mV in $\mathrm{Vm}$ in Iso-treated $D m d^{m d x}$ cardiomyocytes compared with WT cells. The latter suggests that $\mathrm{Cx} 43$ hemichannel open probability (and/or conductance) is only slightly increased by Iso treatment. Greater increase in 
open probability of remodeled Cx43 hemichannels could lead to complete collapse of the electrochemical gradient and cell death (44). Cx43 hemichannel function is tightly regulated by physiological extracellular calcium concentrations (45). In line with this, we found that changes in $V m$ in oocytes expressing $\mathrm{Cx} 43$ strongly correlated with extracellular calcium concentrations (Figure 6D). Thus, extracellular calcium concentrations in our experiments could limit changes in $V m$ in $D m d^{m d x}$ cardiomyocytes treated with Iso.

It has been widely reported that Iso treatment in isolated cardiomyocytes promotes posttranslational modification (i.e., phosphorylation and S-nitrosylation) of various other cardiac ion channels and transporters, which consequently affect the kinetics of the APs $(46,47)$. Interestingly, we observed that Iso-induced AP prolongation in both WT and $D m d^{m d x}$ cardiomyocytes was inhibited by Gap19 and AbCx43, suggesting a role for $\mathrm{Cx} 43$ hemichannels in sustaining AP prolongation (Supplemental Figure 8). Interestingly, unpaired $\mathrm{Cx} 43$ hemichannels are found at the intercalated disc prior to formation of gap junctions in a region known as the perinexus (48-50); thus, it is possible that $\mathrm{Cx} 43$ hemichannels play a physiological role in setting the local membrane potential upon physiological conditions. In pathology, however, overexpression and lateralization may exacerbate their activity and create DADs and TAs. Further experiments are necessary to investigate.

When we assessed $\mathrm{K}^{+}$currents mediated mainly by voltage-gated potassium and inwardly rectifying $\mathrm{K}^{+}$ currents (51-53), these last currents being those that maintain the $V m$ in several cardiac cells (51, 52, 54, 55), WT and $D m d^{m d x}$ cardiomyocytes displayed similar reversal $\mathrm{Vm}$ in the presence or absence of Iso (Supplemental Figure 7B). In addition, Gap19 did not alter $\mathrm{K}^{+}$current reversal potentials in WT and $D m d^{m d x}$ cardiomyocytes. These findings suggest that $\mathrm{K}^{+}$channels did not mediate changes in the resting $V m$ detected in WT and $D m d^{m d x}$ cardiomyocytes upon $\beta$-adrenergic stress.

Recent studies indicate that $\beta$-adrenergic signaling promotes NO production in cardiomyocytes (29, 56-58). In addition to the canonical pathway of NO-induced protein kinase G activation, NO also induces direct posttranslational modification of thiol groups in specific cysteine residues on various proteins via S-nitrosylation (59). S-nitrosylation of several $\mathrm{Ca}^{2+}$-handling proteins, including RyR, SERCA2-associated phospholamban, NCX, and troponin C $(29,30,60)$, are promoted by $\beta$-adrenergic receptors' activation. In the DMD heart, the expression of neuronal NO synthases (nNOSs) and endothelial NO synthases (eNOSs) is reduced (61); however, there is a significant increase in the levels of inducible NO synthase (iNOS) $(10,61)$. This can result in a significant increase in NO production because the iNOS catalytic activity is 100-1000 times higher than that of eNOS and nNOS (62). Several studies suggest that the S-nitrosylation-redox balance might play a key role in ventricular arrhythmias in $D m d^{m d x}$ mice $(10,63,64)$. In particular, Fauconnier et al. showed that RyR2 channels from $D m d^{m d x}$ hearts were S-nitrosylated and depleted of calstabin2 (FKBP12.6), resulting in "leaky" RyR2 channels and increased diastolic sarcoplasmic reticulum (SR) $\mathrm{Ca}^{2+}$ leak. Inhibition of SR $\mathrm{Ca}^{2+}$ leak prevents membrane depolarization, DADs, and arrhythmias in dystrophic mice (10). The molecular mediators by which leaky RyRs promote membrane depolarization in dystrophic cardiomyocytes have not been investigated. Recent work indicates that an increase in intracellular $\mathrm{Ca}^{2+}$ concentration (up to $\sim 500 \mathrm{nM}$ ) activates $\mathrm{Cx} 43$ hemichannels in both heterologous expression systems and ventricular cardiomyocytes (65). Combined with our results, these findings suggest a causal link between increased $\mathrm{SR} \mathrm{Ca}{ }^{2+}$ leak and $\mathrm{Cx} 43$ hemichannel activation in mediating membrane depolarization and arrhythmias in Iso-treated $D m d^{m d x}$ cardiomyocytes and mice, respectively.

S-nitrosylation may also be a potential regulator of Cx43 hemichannels in $D m d^{m d x}$ cardiomyocytes. We found that the level of S-nitrosylated Cx43 was substantially higher in Dmdmdx compared with WT hearts. Under resting conditions, $D m d^{m d x}$ and WT cardiomyocytes displayed S-nitrosylated Cx43 proteins mainly at the intercalated disc regions, where they likely formed gap junction channels. Upon stimulation with Iso, most of the S-nitrosylated Cx43 was found at lateralized regions of $D m d^{m d x}$ cardiomyocytes, where Cx43 is remodeled and primarily forming hemichannels. In contrast, S-nitrosylated $\mathrm{Cx} 43$ remained at the discs in WT cells. Consistent with a possible pathophysiological role of S-nitrosylated Cx43 in DMD, hearts from patients with DMD displayed substantially higher levels of S-nitrosylated Cx43 compared with hearts of healthy individuals. Moreover, whereas S-nitrosylated Cx43 molecules were mostly lateralized in DMD cardiac tissue, they were confined to intercalated discs in hearts of healthy humans.

Inhibition of NO production with L-NAME prevented Iso-induced S-nitrosylation of Cx43 protein. It also prevented increases in membrane permeability and excitability in $D m d^{m d x}$ cardiomyocytes, which is in agreement with an absence of arrhythmogenic behavior in $D m d^{m d x}$ mice treated with Iso. To our knowledge, there is no previous evidence indicating that NO inhibition attenuates cardiac stress-induced arrhythmias in dystrophic mice. 
We propose that an increase in NO production and consequent S-nitrosylation of lateralized Cx43 hemichannels trigger arrhythmias in $D m d^{m d x}$ mice. In line with this, exogenous administration of NO promoted membrane depolarization and TAs in $D m d^{m d x}$ but not in WT cardiomyocytes via activation of Cx43 hemichannels. Importantly, we also rule out that, in addition to S-nitrosylation of Cx43, the canonical NO/GMPc/PKG pathway is involved in the activation of $\mathrm{Cx} 43$ hemichannels. For example, we found that membrane depolarization and TAs in $D m d^{m d x}$ were not affected by ODQ and KT 5823, an inhibitor of soluble guanylyl cyclase and protein kinase G, respectively (Supplemental Figure 9A). Consistently, ODQ or KT 5823 did not affect NO-induced Cx43 hemichannel currents in Xenopus oocytes expressing Cx43 (Supplemental Figure 9B).

Electrophysiological studies of hemichannels in Xenopus oocytes confirmed that NO promotes their opening, and consequently, leads to membrane depolarization. At the molecular level, we found that the Cx43 CT domain is critical for the modification of remodeled hemichannel gating by NO. We identified residue $\mathrm{C} 271$ located within this domain as the unique S-nitrosylation site and for the first time to our knowledge demonstrated that S-nitrosylation of C271 is critical for NO-induced Cx43 hemichannel opening. This is consistent with a previous report identifying $\mathrm{C} 271$ as the site for nitrosylation of $\mathrm{Cx} 43$-forming gap junction channels.

Cx43 remodeling is observed in multiple cardiac pathologies, including ischemia, myocardial infarction, and hypertrophy, and has been linked to arrhythmogenesis because of the reduction of electrical coupling through gap junctions $(17,67-72)$. It is proposed that reduced electrical coupling slows conduction and favors reentrant excitation, resulting in arrhythmias (67-72). However, this is not likely to underlie arrhythmic behaviors observed in DMD hearts, where there is no reduction in total $\mathrm{Cx} 43$ protein levels (and thus coupling) detected (18). Biotin assay experiments suggest that remodeled Cx43 proteins found in dystrophic hearts form undocked hemichannels and not de novo gap junction channels. Compelling evidence indicates that biotin does not bind to $\mathrm{Cx} 43$ molecules that are part of gap junctions $(31,73)$. Furthermore, functional and stable $\mathrm{Cx} 43$ gap junction channels require formation of a molecular complex consisting of several intracellular binding proteins, which were not found to be in association with lateralized Cx43 protein (48). Thus, remodeled lateralized Cx43 hemichannels rather than $\mathrm{Cx} 43$ junctional proteins are likely playing a substantial role in DMD arrhythmogenesis.

Overall, by supporting a role for $\mathrm{Cx} 43$ hemichannels in the development of arrhythmias, our results suggest a major impact of $\mathrm{Cx} 43$ remodeling in other cardiac pathologies, including myocardial infarction and hypertrophy. Cx43 hemichannels emerge as new players that critically affect membrane excitability of $D m d^{m d x}$ cardiomyocytes and promote arrhythmias during $\beta$-adrenergic-induced cardiac stress. Thus, $\mathrm{Cx} 43 \mathrm{hemichan}-$ nels could serve as a novel therapeutic target that can prevent cardiac arrhythmias and heart dysfunction.

\section{Methods}

Cell isolation. Ventricular myocytes were enzymatically isolated from mouse hearts (WT and $D m d^{m d x}$ ). WT and $D m d^{m d x}$ male mice were purchased from The Jackson Laboratory and analyzed at time points of 5-6 months. Mice were heparinized (5000 U/kg) and then anesthetized with overdosed isoflurane. The hearts were removed and were retrogradely perfused at $37^{\circ} \mathrm{C}$ in Langendorff fashion with nominally $\mathrm{Ca}^{2+}$-free Tyrode's solution containing $0.5 \mathrm{mg} / \mathrm{mL}$ collagenase (type II; Worthington) and $0.1 \mathrm{mg} / \mathrm{mL}$ protease (type XIV; MilliporeSigma) for 10 minutes. The $\mathrm{Ca}^{2+}$-free Tyrode's solution contained (in $\mathrm{mM}$ ) $136 \mathrm{NaCl}, 5.4$ $\mathrm{KCl}, 0.33 \mathrm{Na}_{2} \mathrm{PO}_{4}, 1 \mathrm{MgCl}_{2}, 10$ glucose, and $10 \mathrm{HEPES}$ (pH 7.4, adjusted with $\mathrm{NaOH}$ ). The enzyme solution was then washed out, and the hearts were removed from the perfusion apparatus. Left ventricles were placed in Petri dishes and were gently teased apart with forceps. Then, the cardiomyocytes were filtered through nylon mesh. The $\mathrm{Ca}^{2+}$ concentration was gradually increased to $1.0 \mathrm{mM}$, and the cells were stored at room temperature and used within 8 hours. Only cells from the left ventricular wall were used.

Electrophysiology. Isolated cardiomyocytes were patch-clamped using the whole-cell configuration of the patch-clamp technique in the current-clamp or the voltage-clamp mode. To record APs, patch pipettes (2-5 M $\Omega$ ) were filled with an internal solution containing (in $\mathrm{mM}$ ) $110 \mathrm{~K}^{+}$-aspartate, $30 \mathrm{KCl}, 5 \mathrm{NaCl}, 10$ HEPES, 0.1 EGTA, $5 \mathrm{Mg}$-ATP, and $5 \mathrm{Na}_{2}$-creatine phosphate ( $\mathrm{pH} 7.2$, adjusted with $\mathrm{KOH}$ ). The myocytes were superfused with normal Tyrode's solution containing (in $\mathrm{mM}$ ) $136 \mathrm{NaCl}, 5.4 \mathrm{KCl}, 0.33 \mathrm{Na}_{2} \mathrm{PO}_{4}, 1.0$ $\mathrm{CaCl}_{2}, 1 \mathrm{MgCl}_{2}, 10$ glucose, and $10 \mathrm{HEPES}$ ( $\mathrm{pH} 7.4$, adjusted with $\mathrm{NaOH}$ ). APs were elicited with 2-ms, 2- to 4-nA square pulses at various pacing cycle lengths. We quantified TAs and changes in the resting $V m$ induced by Iso between 5 and 10 minutes after stimulation. The Gap19 peptide $(232 \mathrm{ng} / \mu \mathrm{L})$ and $\mathrm{Cx} 43$ antibody $(2.5 \mathrm{ng} / \mu \mathrm{L})$ were added in the pipette solution to block $\mathrm{Cx} 43$ hemichannel activity. We used between 2 and 3 cardiomyocytes per isolated heart per condition. 
The 2-electrode voltage clamp technique and Xenopus oocytes were used to test hemichannel currents from homomeric and heteromeric channels formed by hCx43, hCx26, and hCx26S17F. Oocytes were enzymatically obtained from ovaries of female Xenopus laevis purchased from Xenopus Express. All connexin clones were purchased from Origene. Nhe1-linearized $\mathrm{hCx} 43, \mathrm{hCx} 26$, and $\mathrm{hCx} 26 \mathrm{~S} 17 \mathrm{~F}$ DNA were transcribed in vitro to cRNAs using the T7 Message Machine kit (Ambion). Electrophysiological data were collected using pCLAMP10 software (Molecular Devices Corporation). All recordings were made at room temperature $\left(20^{\circ} \mathrm{C}-22^{\circ} \mathrm{C}\right)$. For $\mathrm{Cx} 43$-expressing oocytes, the recording solutions contained (in $\left.\mathrm{mM}\right) 117$ tetraethylammonium (TEA) and 5 HEPES and $0.2 \mathrm{mM}$ extracellular $\mathrm{Ca}^{2+}$ concentration (pH 7.4, adjusted with $\mathrm{N}$-methyl-D-glucamine). The recording solutions for $\mathrm{Cx} 43 \mathrm{Cx} 26 \mathrm{~S} 17 \mathrm{~F}$ heteromeric hemichannels contained (in $\mathrm{mM}$ ) $118 \mathrm{NaCl}$, $2 \mathrm{KCl}$, and $5 \mathrm{HEPES}$ and $1.8 \mathrm{mM}$ of extracellular $\mathrm{Ca}^{2+}$ concentration ( $\mathrm{pH} 7.4$, adjusted with $\mathrm{NaOH}$ ). Currents from oocytes were recorded 2 days after cRNA injection, using a Warner OC-725 amplifier (Warner Instruments). Currents were sampled at $2 \mathrm{kHz}$ and low-pass filtered as $0.5 \mathrm{kHz}$. Microelectrode resistances were between 0.1 and $1.2 \mathrm{M} \Omega$ when filled with $3 \mathrm{M} \mathrm{KCl}$. Antisense oligonucleotides against $\mathrm{Cx} 38$ were injected into each oocyte to reduce the expression of endogenous $\mathrm{Cx} 38$ at 4 hours after harvesting the oocytes $(1 \mathrm{mg} / \mathrm{mL}$; using the sequence from Ebihara [ref. 74]). We assessed hemichannel currents and changes in the resting $V m$ evoked by $\mathrm{NO}$ at 10 minutes after stimulation. We used at least 3 oocytes per each independent frog.

Electrocardiography. Whole-animal ECGs were recorded using needle electrodes in a lead II conformation. Animals were anesthetized by Avertin (2,2,2-tribromoethanol, $290 \mathrm{mg} / \mathrm{kg} \mathrm{IP}$ ) and kept at a constant $37^{\circ} \mathrm{C}$ temperature using a heating pad for the duration of analysis. For inhibition of NO production, animals were treated for 1 week with $2 \mathrm{mM}$ L-NAME (MilliporeSigma) via water solution. Following treatment time, mice were tested with Iso (5 mg/ $\mathrm{kg}$ IP). ECGs were recorded before (baseline line) and after Iso treatment. Arrhythmias were scored based on a point system: 0 , no arrhythmias; 1 , single PVCs; 2, double PVCs; 3 , nonsustained VT; 4, sustained VT or AV block; and 5, death. We used between 6 and 7 independent mice per experiment.

Dye perfusion and uptake in isolated hearts. Mice were heparinized (5000 U/ kg) and then anesthetized with Avertin (2,2,2-tribromoethanol, $290 \mathrm{mg} / \mathrm{kg} \mathrm{IP}$ ). Once unconscious, mice were injected with either saline (control) or Iso ( $5 \mathrm{mg} / \mathrm{kg}$ IP). Twenty minutes following Iso or vehicle injection, mice were sacrificed, and hearts were extracted and cannulated in a Langendorff perfusion system. Hearts were perfused with normal Tyrode's buffer (NT) (in mM: $136 \mathrm{NaCl}, 5.4 \mathrm{KCl}, 0.33 \mathrm{NaH}_{2} \mathrm{PO}_{4}, 1 \mathrm{MgCl}_{2}$, $1 \mathrm{CaCl}_{2}, 10 \mathrm{HEPES}$, and 10 glucose $)$ at $37^{\circ} \mathrm{C}$ for 10 minutes, NT containing ethidium bromide $(5 \mu \mathrm{M})$ or PI $(50 \mu \mathrm{M})$ for 20 minutes, and then NT buffer for 5 minutes to wash out the dye. Hearts were then fixed overnight in 4\% paraformaldehyde (MilliporeSigma), placed into 30\% sucrose solution in PBS (MilliporeSigma) for 12 hours, then embedded in O.C.T. (Tissue-Tek, Sakura Finetek). Subsequently $10-\mu \mathrm{m}$ cryosections were made; slides were thawed to room temperature and washed in PBS, and Alexa Fluor 488 Wheat Germ Agglutinin (Invitrogen, Thermo Fisher Scientific) was applied for 20 minutes. Slides were then washed in PBS and mounted with mounting reagent with DAPI (Invitrogen, Thermo Fisher Scientific). Slides were then imaged using a 200 Axiovert fluorescence microscope (Zeiss). To calculate ethidium fluorescence in ImageJ (NIH), DAPI-stained nuclei were identified and created as regions of interest (ROIs), and individual nuclei (100-150 per image) mean fluorescence intensities were measured. Then, the ROI outlines were projected onto the corresponding ethidium image, where individual fluorescence intensities were measured, capturing ethidium signal within all nuclei. Ethidium intensity was then divided by DAPI nuclei intensity per respective ROI signal; then the mean ratio was calculated for all nuclei in the image. We used 6 independent hearts per experiment. In addition, 3 images per heart were evaluated in a blinded manner.

Biotin perfusion of isolated hearts. Mice were heparinized and then anesthetized with Avertin (2,2,2-tribromoethanol, $290 \mathrm{mg} / \mathrm{kg} \mathrm{IP}$ ). Once unconscious, mice were sacrificed, and hearts were extracted and cannulated in a Langendorff perfusion system. Hearts were initially perfused with NT for 5 minutes, switched to NT buffer biotin (EZ-Link NHS biotin, $0.5 \mathrm{mg} / \mathrm{mL}$, Thermo Fisher Scientific) for 60 minutes $(0.25 \mathrm{~mL} /$ min flow rate), and washed out for 10 minutes with NT buffer plus $15 \mathrm{mM}$ glycine. Left ventricular tissue was then homogenized in HEN buffer (in mM: 250 HEPES, 1 EDTA, and 0.1 neocuproine, $\mathrm{pH} 7.7$ ) with $2 \times$ HALT protease inhibitors (Thermo Fisher Scientific) and then centrifuged at 16,000 $\mathrm{g}$ for 10 minutes. Following protein concentration determination, $50 \mu \mathrm{L}$ streptavidin beads (Thermo Fisher Scientific) were added to $200 \mu \mathrm{g}$ protein and nutated for 90 minutes at $4^{\circ} \mathrm{C}$ with occasional vortexing. Samples were then centrifuged at 16,000 $\mathrm{g}$ for 2 minutes, and the supernatant was discarded. The streptavidin pellet was then resuspended in fresh lysis buffer containing $0.1 \%$ Triton X-100 and centrifuged for 1 minute at 16,000 g. 
The pellet was then washed with PBS ( $\mathrm{pH} 7.4$ ) and centrifuged. Next, $25 \mu \mathrm{L}$ of $2 \times$ Laemmli sample buffer was added and heated at $100^{\circ} \mathrm{C}$ for 5 minutes to disrupt biotin-streptavidin interaction. The heated samples were then centrifuged for 1 minute at $16,000 \mathrm{~g}$, and the supernatant was run along with total protein extracts without streptavidin pulldown on SDS-PAGE.

Immunofluorescence. Mouse ventricular tissue was frozen in O.C.T. (Tissue-Tek, Sakura Finetek). Cryosections were cut at $6 \mu \mathrm{m}$; slides were thawed to room temperature, washed in PBS for 10 minutes, and blocked for 1 hour at room temperature with 10\% normal donkey serum (Jackson Immunoresearch) in PBSt wash buffer (PBS $+0.1 \%$ Tween-20). Sections were then incubated with both $\mathrm{N}$-cadherin (Invitrogen, Thermo Fisher Scientific, 33-3900, 1:200, mouse) and biotin (Abcam, ab53494, 1:200, rabbit) antibodies in blocking buffer overnight at $4^{\circ} \mathrm{C}$. Following 3 washes in PBSt, sections were incubated for an hour at room temperature with Alexa Fluor-conjugated secondary antibodies (Jackson Immunoresearch) in blocking buffer (1:200). Slides were subsequently washed in PBSt, and coverslips were mounted using ProLong gold antifade reagent containing WGA, Alexa Fluor 350 Conjugate (Invitrogen, Thermo Fisher Scientific). Sections were imaged on a 200 Axiovert fluorescence microscope (Zeiss).

Western blotting. Protein samples from the left ventricular heart wall or from injected Xenopus oocytes were separated by $10 \%$ SDS-PAGE and transferred onto a PVDF membrane (Bio-Rad). The primary Cx43 (MilliporeSigma, C8093, 1:2000, mouse), eNOS (Thermo Fisher Scientific, 9D10, 1:1000, mouse), and N-cadherin (Invitrogen, Thermo Fisher Scientific, 33-3900, 1:2000, mouse) antibodies and secondary antibodies (Pierce Biotechnology, Thermo Fisher Scientific, 1/5000) were incubated using the Signal Enhancer HIKARI (Nacalai Tesque, Inc). The protein bands were detected with the SuperSignal West Femto (Pierce Biotechnology, Thermo Fisher Scientific). Molecular mass was estimated with prestained markers (Bio-Rad). Protein bands were analyzed using ImageJ software (NIH). We used 6 independent hearts per treatment.

Detection of S-nitrosylated proteins. S-nitrosylated proteins were isolated from either mice heart ventricular samples or Xenopus oocytes expressing Cx43 WT or mutant hemichannels. Heart tissue and Xenopus oocytes were homogenized in HEN buffer (in mM: 250 HEPES, 1 EDTA, and 0.1 neocuproine, $\mathrm{pH}$ 7.7) containing protease inhibitors. Samples containing $200 \mu \mathrm{g}$ protein were treated by the biotin switch method to pull down all S-nitrosylated proteins (75). Briefly, samples were incubated with methyl methanethiosulfonate reagents (MMTS, MilliporeSigma) for 1 hour at $50^{\circ} \mathrm{C}$ in the dark to block cysteine-free thiols. Afterward, proteins were precipitated with 4 volumes of ice-cold acetone, repeatedly washed with acetone to remove free MMTS, and resolubilized. Thereafter, nitrosylated cysteine residues were reduced to free cysteine by incubating 1 hour with $30 \mathrm{mM}$ sodium ascorbate (MilliporeSigma) and labeled with HPDP-biotin (Thermo Fisher Scientific). Proteins were precipitated with acetone to wash the excess of HPDP-biotin and solubilized for Western blot analysis. Following solubilization, the samples were incubated 1 hour with agarose-conjugated streptavidin beads (Thermo Fisher Scientific) and centrifuged to pull down HPDP-biotinylated proteins. Adsorbed proteins were separated using 10\% SDS-PAGE and transferred onto a PVDF membrane (Bio-Rad). A monoclonal anti-Cx43 (MilliporeSigma, C8093, 1:2000, mouse) was used to detect Cx43 protein. For all Western blot analysis, the intensity of the signal was evaluated using the ImageJ program. We used 6 independent hearts per treatment.

Analysis of protein-to-protein association. The subcellular distribution and possible spatial association between S-nitrosylation and Cx43 were evaluated by PLA (76) (MilliporeSigma). Tissue sections (6 $\mu \mathrm{m})$ were blocked and incubated with 2 primary antibodies from different species, which were, then, detected using oligonucleotide-conjugated secondary antibodies as described in the manufacturer's protocols. The antibodies used were a monoclonal anti-Cx43 (MilliporeSigma, C8093, 1:200) and an anti-S-nitrosocysteine antibody (MilliporeSigma, N5411, 1:100). If the target proteins were closer than $20 \mathrm{~nm}$, the oligonucleotides could be used as templates for DNA ligase-mediated joining of additional oligonucleotides to form a circular DNA molecule, which was amplified using hybridizing fluorophore-labeled oligonucleotides. Images were visualized with a 200 Axiovert fluorescence microscope (Zeiss). We used 5 independent hearts per treatment.

Chemicals. L-NAME, HEPES, cAMP, $\mathrm{Na}_{2}$-creatine phosphate, $\mathrm{K}^{+}$-aspartate, $N$-methyl-D-glucamine, and TEA were purchased from MilliporeSigma. DEENO and Iso were obtained from Calbiochem and collagenase type II from Worthington. Gap19 was purchased from Tocris.

Statistics. Values are displayed as mean \pm standard error of the mean. Comparisons between groups were made using 1-way ANOVA or 2-way ANOVA plus Tukey's post hoc test, as appropriate. In the case of 2 groups, we performed paired 2-tailed Student's $t$ test. $P<0.05$ was considered significant. Each figure indicates the respective $n$ value. 
Study approval. All animal experiments were approved by the IACUC of Rutgers New Jersey Medical School and performed in accordance with the NIH guidelines.

Two non-DMD and 2 DMD male human heart samples were obtained from the University of Maryland Brain and Tissue Bank, a member of the NIH NeuroBioBank network. All samples were dissected postmortem. Informed consent was obtained from all subjects from whom tissues were analyzed. All human experiments were approved by the IRB of Rutgers University (Newark, New Jersey, USA) and performed in accordance with relevant guidelines and regulations.

\section{Author contributions}

MAL, EH, DF, NS, LHX, and JEC designed experiments. MAL performed most of the experiments. MAL, JEC, LHX, and NS analyzed the data. MAL and JEC wrote the manuscript. EH and NS edited the manuscript. All authors reviewed and approved the final draft.

\section{Acknowledgments}

We thank Theanne Griffith for critically reading the manuscript. This work was supported by an American Heart Association (AHA) postdoctoral fellowship 18POST339610107 to MAL; AHA predoctoral fellowship 17PRE33660354 to EH; Muscular Dystrophy Association grant 416281 to DF; NIH grant HL093342 to NS; NIH grants R01HL92929 and R01H1133294 to LHX; AHA grant 16GRNT31100022 to LHX; NIH grant R01HL141170 to DF, NS, and JEC; and NIH grant R01GM099490 to JEC.

Address correspondence to: Jorge E. Contreras; Medical Sciences Building I-615, 185 S. Orange Avenue, Newark, New Jersey 07103, USA. Phone: 973-972-3666; Email: contrejo@njms.rutgers.edu.

1. Davies KE, Nowak KJ. Molecular mechanisms of muscular dystrophies: old and new players. Nat Rev Mol Cell Biol. 2006;7(10):762-773.

2. Hoffman EP, Brown RH, Kunkel LM. Dystrophin: the protein product of the Duchenne muscular dystrophy locus. Cell. 1987;51(6):919-928

3. de Kermadec JM, Bécane HM, Chénard A, Tertrain F, Weiss Y. Prevalence of left ventricular systolic dysfunction in Duchenne muscular dystrophy: an echocardiographic study. Am Heart J. 1994;127(3):618-623.

4. Connuck DM, et al. Characteristics and outcomes of cardiomyopathy in children with Duchenne or Becker muscular dystrophy: a comparative study from the Pediatric Cardiomyopathy Registry. Am Heart J. 2008;155(6):998-1005.

5. Hermans MC, Pinto YM, Merkies IS, de Die-Smulders CE, Crijns HJ, Faber CG. Hereditary muscular dystrophies and the heart. Neuromuscul Disord. 2010;20(8):479-492.

6. Bushby K, et al. Diagnosis and management of Duchenne muscular dystrophy, part 2: implementation of multidisciplinary care. Lancet Neurol. 2010;9(2):177-189.

7. Garg R, Yusuf S. Overview of randomized trials of angiotensin-converting enzyme inhibitors on mortality and morbidity in patients with heart failure. Collaborative Group on ACE Inhibitor Trials. JAMA. 1995;273(18):1450-1456.

8. Ather S, Wang W, Wang Q, Li N, Anderson ME, Wehrens XH. Inhibition of CaMKII phosphorylation of RyR2 prevents inducible ventricular arrhythmias in mice with Duchenne muscular dystrophy. Heart Rhythm. 2013;10(4):592-599.

9. Kyrychenko S, et al. Hierarchical accumulation of RyR post-translational modifications drives disease progression in dystrophic cardiomyopathy. Cardiovasc Res. 2013;97(4):666-675.

10. Fauconnier J, et al. Leaky RyR2 trigger ventricular arrhythmias in Duchenne muscular dystrophy. Proc Natl Acad Sci USA. 2010;107(4):1559-1564.

11. Kleber AG, Saffitz JE. Role of the intercalated disc in cardiac propagation and arrhythmogenesis. Front Physiol. $2014 ; 5: 404$.

12. Gros DB, Jongsma HJ. Connexins in mammalian heart function. Bioessays. 1996;18(9):719-730.

13. Severs NJ, Coppen SR, Dupont E, Yeh HI, Ko YS, Matsushita T. Gap junction alterations in human cardiac disease. Cardiovasc Res. 2004;62(2):368-377.

14. Severs NJ, et al. Remodelling of gap junctions and connexin expression in heart disease. Biochim Biophys Acta. 2004;1662(1-2):138-148.

15. Severs NJ, et al. Alterations in cardiac connexin expression in cardiomyopathies. Adv Cardiol. 2006;42:228-242.

16. Gonzalez JP, Ramachandran J, Xie LH, Contreras JE, Fraidenraich D. Selective Connexin43 inhibition prevents isoproterenol-induced arrhythmias and lethality in muscular dystrophy mice. Sci Rep. 2015;5:13490.

17. Colussi C, et al. Ne-lysine acetylation determines dissociation from GAP junctions and lateralization of connexin 43 in normal and dystrophic heart. Proc Natl Acad Sci USA. 2011;108(7):2795-2800.

18. Gonzalez JP, et al. Normalization of connexin 43 protein levels prevents cellular and functional signs of dystrophic cardiomyopathy in mice. Neuromuscul Disord. 2018;28(4):361-372.

19. Abudara V, et al. The connexin43 mimetic peptide Gap19 inhibits hemichannels without altering gap junctional communication in astrocytes. Front Cell Neurosci. 2014;8:306.

20. Wang N, et al. Selective inhibition of Cx43 hemichannels by Gap19 and its impact on myocardial ischemia/reperfusion injury. Basic Res Cardiol. 2013;108(1):309.

21. Hegyi B, et al. Enhanced depolarization drive in failing rabbit ventricular myocytes. Circ Arrhythm Electrophysiol. 2019;12(3):e007061 
22. Hegyi B, et al. Complex electrophysiological remodeling in postinfarction ischemic heart failure. Proc Natl Acad Sci USA 2018;115(13):E3036-E3044.

23. Sato D, Clancy CE, Bers DM. Dynamics of sodium current mediated early afterdepolarizations. Heliyon. 2017;3(9):e00388.

24. Baczkó I, Giles WR, Light PE. Resting membrane potential regulates $\mathrm{Na}(+)-\mathrm{Ca} 2+$ exchange-mediated Ca2+ overload during hypoxia-reoxygenation in rat ventricular myocytes. $J$ Physiol (Lond). 2003;550(Pt 3):889-898.

25. Matsuda T, et al. SEA0400, a novel and selective inhibitor of the $\mathrm{Na}+\mathrm{Ca} 2+$ exchanger, attenuates reperfusion injury in the in vitro and in vivo cerebral ischemic models. J Pharmacol Exp Ther. 2001;298(1):249-256.

26. Contreras JE, Sáez JC, Bukauskas FF, Bennett MV. Gating and regulation of connexin 43 (Cx43) hemichannels. Proc Natl Acad Sci USA. 2003;100(20):11388-11393.

27. Johnson RG, et al. Connexin hemichannels: methods for dye uptake and leakage. JMembr Biol. 2016;249(6):713-741.

28. Figueroa XF, Lillo MA, Gaete PS, Riquelme MA, Sáez JC. Diffusion of nitric oxide across cell membranes of the vascular wall requires specific connexin-based channels. Neuropharmacology. 2013;75:471-478.

29. Irie T, et al. S-Nitrosylation of Calcium-handling proteins in cardiac adrenergic signaling and hypertrophy. Circ Res. 2015;117(9):793-803.

30. Vielma AZ, León L, Fernández IC, González DR, Boric MP. Nitric oxide synthase 1 modulates basal and $\beta$-adrenergic-stimulated contractility by rapid and reversible redox-dependent S-nitrosylation of the heart. PLoS ONE. 2016;11(8):e0160813.

31. Retamal MA, Cortés CJ, Reuss L, Bennett MV, Sáez JC. S-nitrosylation and permeation through connexin 43 hemichannels in astrocytes: induction by oxidant stress and reversal by reducing agents. Proc Natl Acad Sci USA. 2006;103(12):4475-4480.

32. Pfeiffer S, Leopold E, Schmidt K, Brunner F, Mayer B. Inhibition of nitric oxide synthesis by NG-nitro-L-arginine methyl ester (L-NAME): requirement for bioactivation to the free acid, NG-nitro-L-arginine. Br J Pharmacol. 1996;118(6):1433-1440.

33. García IE, et al. Keratitis-ichthyosis-deafness syndrome-associated Cx26 mutants produce nonfunctional gap junctions but hyperactive hemichannels when co-expressed with wild type Cx43. J Invest Dermatol. 2015;135(5):1338-1347.

34. Hansen DB, Braunstein TH, Nielsen MS, MacAulay N. Distinct permeation profiles of the connexin 30 and 43 hemichannels. FEBS Lett. 2014;588(8):1446-1457.

35. White TW, et al. Functional characteristics of skate connexin35, a member of the gamma subfamily of connexins expressed in the vertebrate retina. Eur J Neurosci. 1999;11(6):1883-1890.

36. Srinivas M, et al. Connexin43 mutations linked to skin disease have augmented hemichannel activity. Sci Rep. 2019;9(1):19.

37. Lampe PD, Lau AF. The effects of connexin phosphorylation on gap junctional communication. Int J Biochem Cell Biol. 2004;36(7):1171-1186

38. Laird DW, Puranam KL, Revel JP. Turnover and phosphorylation dynamics of connexin43 gap junction protein in cultured cardiac myocytes. Biochem J. 1991;273(Pt 1):67-72.

39. Solan JL, Lampe PD. Specific Cx43 phosphorylation events regulate gap junction turnover in vivo. FEBS Lett. 2014;588(8):1423-1429.

40. Marban E, Robinson SW, Wier WG. Mechanisms of arrhythmogenic delayed and early afterdepolarizations in ferret ventricular muscle. J Clin Invest. 1986;78(5):1185-1192.

41. Priori SG, Corr PB. Mechanisms underlying early and delayed afterdepolarizations induced by catecholamines. Am J Physiol. 1990;258(6 Pt 2):H1796-H1805.

42. Priori SG, Mantica M, Napolitano C, Schwartz PJ. Early afterdepolarizations induced in vivo by reperfusion of ischemic myocardium. A possible mechanism for reperfusion arrhythmias. Circulation. 1990;81(6):1911-1920.

43. Lin $\mathrm{X}$, et al. Beta-adrenergic stimulation does not activate $\mathrm{Na}+/ \mathrm{Ca} 2+$ exchange current in guinea pig, mouse, and rat ventricular myocytes. Am J Physiol, Cell Physiol. 2006;290(2):C601-C608.

44. Laird DW, Lampe PD. Therapeutic strategies targeting connexins. Nat Rev Drug Discov. 2018;17(12):905-921.

45. Lopez W, Ramachandran J, Alsamarah A, Luo Y, Harris AL, Contreras JE. Mechanism of gating by calcium in connexin hemichannels. Proc Natl Acad Sci USA. 2016;113(49):E7986-E7995.

46. Gómez R, et al. Nitric oxide increases cardiac IK1 by nitrosylation of cysteine 76 of Kir2.1 channels. Circ Res. 2009;105(4):383-392.

47. Tamargo J, Caballero R, Gómez R, Delpón E. Cardiac electrophysiological effects of nitric oxide. Cardiovasc Res. 2010;87(4):593-600

48. Rhett JM, Gourdie RG. The perinexus: a new feature of Cx43 gap junction organization. Heart Rhythm. 2012;9(4):619-623

49. Rhett JM, Veeraraghavan R, Poelzing S, Gourdie RG. The perinexus: sign-post on the path to a new model of cardiac conduction? Trends Cardiovasc Med. 2013;23(6):222-228.

50. Hoagland DT, Santos W, Poelzing S, Gourdie RG. The role of the gap junction perinexus in cardiac conduction: potential as a novel anti-arrhythmic drug target. Prog Biophys Mol Biol. 2019;144:41-50.

51. Chilton $\mathrm{L}$, et al. $\mathrm{K}+$ currents regulate the resting membrane potential, proliferation, and contractile responses in ventricular fibroblasts and myofibroblasts. Am J Physiol Heart Circ Physiol. 2005;288(6):H2931-H2939.

52. Vandenberg CA. Inward rectification of a potassium channel in cardiac ventricular cells depends on internal magnesium ions. Proc Natl Acad Sci USA. 1987;84(8):2560-2564.

53. Yuan XJ. Voltage-gated $\mathrm{K}+$ currents regulate resting membrane potential and $[\mathrm{Ca} 2+] \mathrm{i}$ in pulmonary arterial myocytes. Circ Res. 1995;77(2):370-378

54. Miragoli M, Gaudesius G, Rohr S. Electrotonic modulation of cardiac impulse conduction by myofibroblasts. Circ Res. 2006;98(6):801-810.

55. Reimann F, Ashcroft FM. Inwardly rectifying potassium channels. Curr Opin Cell Biol. 1999;11(4):503-508.

56. Curran J, et al. Nitric oxide-dependent activation of CaMKII increases diastolic sarcoplasmic reticulum calcium release in cardiac myocytes in response to adrenergic stimulation. PLoS ONE. 2014;9(2):e87495.

57. Pereira L, Bare DJ, Galice S, Shannon TR, Bers DM. $\beta$-Adrenergic induced SR Ca ${ }^{2+}$ leak is mediated by an Epac-NOS pathway J Mol Cell Cardiol. 2017;108:8-16.

58. Gutierrez DA, Fernandez-Tenorio M, Ogrodnik J, Niggli E. NO-dependent CaMKII activation during $\beta$-adrenergic stimulation of cardiac muscle. Cardiovasc Res. 2013;100(3):392-401.

59. Hess DT, Matsumoto A, Kim SO, Marshall HE, Stamler JS. Protein S-nitrosylation: purview and parameters. Nat Rev Mol Cell 
Biol. 2005;6(2):150-166.

60. Chung HS, et al. Transient receptor potential channel 6 regulates abnormal cardiac S-nitrosylation in Duchenne muscular dystrophy. Proc Natl Acad Sci USA. 2017;114(50):E10763-E10771.

61. Bia BL, et al. Decreased myocardial nNOS, increased iNOS and abnormal ECGs in mouse models of Duchenne muscular dystrophy. J Mol Cell Cardiol. 1999;31(10):1857-1862.

62. MacMicking J, Xie QW, Nathan C. Nitric oxide and macrophage function. Annu Rev Immunol. 1997;15:323-350.

63. Cutler MJ, et al. Aberrant S-nitrosylation mediates calcium-triggered ventricular arrhythmia in the intact heart. Proc Natl Acad Sci USA. 2012;109(44):18186-18191.

64. Gonzalez DR, Beigi F, Treuer AV, Hare JM. Deficient ryanodine receptor S-nitrosylation increases sarcoplasmic reticulum calcium leak and arrhythmogenesis in cardiomyocytes. Proc Natl Acad Sci USA. 2007;104(51):20612-20617.

65. Wang N, et al. Connexin mimetic peptides inhibit Cx43 hemichannel opening triggered by voltage and intracellular Ca2+ elevation. Basic Res Cardiol. 2012;107(6):304.

66. Straub AC, et al. Compartmentalized connexin 43 s-nitrosylation/denitrosylation regulates heterocellular communication in the vessel wall. Arterioscler Thromb Vasc Biol. 2011;31(2):399-407.

67. Yao JA, Hussain W, Patel P, Peters NS, Boyden PA, Wit AL. Remodeling of gap junctional channel function in epicardial border zone of healing canine infarcts. Circ Res. 2003;92(4):437-443.

68. Peters NS, Coromilas J, Severs NJ, Wit AL. Disturbed connexin43 gap junction distribution correlates with the location of reentrant circuits in the epicardial border zone of healing canine infarcts that cause ventricular tachycardia. Circulation. 1997;95(4):988-996.

69. Luke RA, Saffitz JE. Remodeling of ventricular conduction pathways in healed canine infarct border zones. J Clin Invest. 1991;87(5):1594-1602.

70. Kléber AG, Riegger CB, Janse MJ. Electrical uncoupling and increase of extracellular resistance after induction of ischemia in isolated, arterially perfused rabbit papillary muscle. Circ Res. 1987;61(2):271-279.

71. Cooklin M, Wallis WR, Sheridan DJ, Fry CH. Changes in cell-to-cell electrical coupling associated with left ventricular hypertrophy. Circ Res. 1997;80(6):765-771

72. Lin X, Gemel J, Beyer EC, Veenstra RD. Dynamic model for ventricular junctional conductance during the cardiac action potential. Am J Physiol Heart Circ Physiol. 2005;288(3):H1113-H1123.

73. Musil LS, Goodenough DA. Biochemical analysis of connexin43 intracellular transport, phosphorylation, and assembly into gap junctional plaques. J Cell Biol. 1991;115(5):1357-1374.

74. Ebihara L. Xenopus connexin38 forms hemi-gap-junctional channels in the nonjunctional plasma membrane of Xenopus oocytes. Biophys J. 1996;71(2):742-748.

75. Jaffrey SR, Snyder SH. The biotin switch method for the detection of S-nitrosylated proteins. Sci STKE. 2001;2001(86):p11.

76. Söderberg O, et al. Direct observation of individual endogenous protein complexes in situ by proximity ligation. Nat Methods. 2006;3(12):995-1000. 